\title{
Dynamical correlations in the spin-half two-channel Kondo model
}

\author{
A. I. Tóth and G. Zaránd \\ Theoretical Physics Department, Institute of Physics, \\ Budapest University of Technology and Economics, H-1521 Budapest, Hungary
}

(Dated: February 4, 2008)

\begin{abstract}
Dynamical correlations of various local operators are studied in the spin-half two-channel Kondo $(2 \mathrm{CK})$ model in the presence of channel anisotropy or external magnetic field. A conformal field theory-based scaling approach is used to predict the analytic properties of various spectral functions in the vicinity of the two-channel Kondo fixed point. These analytical results compare well with highly accurate density matrix numerical renormalization group results. The universal cross-over functions interpolating between channel-anisotropy or magnetic field-induced Fermi liquid regimes and the two-channel Kondo, non-Fermi liquid regimes are determined numerically. The boundaries of the real $2 \mathrm{CK}$ scaling regime are found to be rather restricted, and to depend both on the type of the perturbation and on the specific operator whose correlation function is studied. In a small magnetic field, a universal resonance is observed in the local fermion's spectral function. The dominant superconducting instability appears in the composite superconducting channel.

PACS numbers: 71.10.Hf, 71.10.Pm, 71.27.+a, 72.15.Qm, 73.43.Nq, 75.20.Hr
\end{abstract}

\section{INTRODUCTION}

Deviations from Fermi liquid-like behavior observed e.g. in the metallic state of high-temperature cuprate superconductors $, 1,2$ or in heavy fermion systems 3,4 prompted physicists to look for new non-Fermi liquid (NFL) compounds. So far a large number of such exotic compounds has been found and investigated. In these systems electrons remain incoherent down to very low temperatures and the usual Fermi liquid description breaks down. To our current understanding, NFL physics may arise in many different ways: it can occur due to some local dynamical quantum fluctuations often described by quantum impurity models,,$\underset{5,6,7}{,}$ it can also be attributed to the presence of the quantum fluctuations of an order parameter or some collective modes, as is the case in the vicinity of many quantum phase transitions $, 6,8$ or for the prototypical example of a Luttinger liquid, $, 9,10,11,12,13$ where electrons are totally disintegrated into collective excitations of the electron gas. NFL physics can also appear as a consequence of disorder like e.g. in disordered Kondo alloys. 14,15

In this paper we study a variant of the overscreened multi-channel Kondo model: the spin- $\frac{1}{2}$, two-channel Kondo (2CK) model, which is the simplest prototypical example of non-Fermi liquid quantum impurity models. This model has first been introduced by Nozières and Blandin, 16 and since has been proposed to describe a variety of systems including dilute heavy fermion compounds,, 5 tunneling impurities in disordered metals and doped semiconductors. 17,18,19 More recently, the 2CK state has been observed in a very controlled way in a double dot system originally proposed by Oreg and Goldhaber-Gordon.20,2149

The two-channel Kondo model consists of a spin- $\frac{1}{2}$, local moment which is coupled through antiferromagnetic exchange interactions to two channels of conduction electrons. Electrons in both channels try to screen the impurity spin. If the coupling of the spin to one of the channels is stronger than to the other then electrons in the more strongly coupled channel screen the spin, while the other channel becomes decoupled. However, for equal exchange couplings, the competition between the two channels leads to overscreening and results in a non-Fermi liquid behavior: Among others, it is characterized by a non-trivial zero temperature residual entropy, a square root-like temperature dependence of the differential conductance, a logarithmic divergence of the spin susceptibility and the linear specific heat coefficient at low temperatures. $\frac{5}{5}$ This unusual and fragile ground state cannot be described within the framework of Nozières' Fermi liquid theory. 25

Being a prototypical example of non-Fermi liquid models, the two-channel Kondo model (2CKM) has already been investigated with a number of methods. These include non-perturbative techniques like the Bethe Ansatz, which gives full account of the thermodynamic properties, $, 26,27$ boundary conformal field theory, $\frac{28}{-}$ which describes the vicinity of the fixed points, and numerical renormalization group (NRG) methods.29 Furthermore other less powerful approximate methods such as the Yuval-Anderson approach, 31 Abelian bosonization, 32 large- $f$ expansion, $, 33,34$ and non-crossing approximation ${ }^{35}$ have also been used to study the 2 CKM successfully.

Rather surprisingly, despite this extensive work, very little is known about dynamical correlation functions such as the spin susceptibility, local charge and superconducting susceptibilities. Even the detailed properties of the $T$-matrix, essential to understand elastic and inelastic scattering in this non-Fermi liquid case, ${ }^{36}$ have only been computed earlier using conformal field theory (which is rather limited in energy range) and by the non-crossing approximation (which is not well-controlled and is unable to describe the Fermi liquid cross-over) ${ }^{37.38}$ It was also possible to compute some of the dynamical correlation functions in case of extreme spin anisotropy us- 


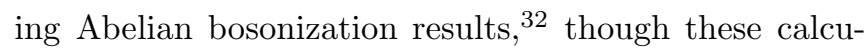
lations reproduce only partly the generic features of the spin-isotropic model ${ }^{39}$ Local correlations in the Anderson model around the non-Fermi liquid fixed point have already been investigated with the use of NRG, although in the absence of channel anisotropy and magnetic field $\stackrel{40}{\underline{4}}$ However, a thorough and careful NRG analysis of the $T=0$ temperature $T$-matrix of the $2 \mathrm{CKM}$ has been carried out only very recently,$\underline{24.36}$ and the $T \neq 0$ analysis still needs to be done.

The main purpose of this paper is to fill this gap by giving a comprehensive analysis of the local correlation functions at zero temperature using the numerical renormalization group approach. However, in the vicinity of the rather delicate two-channel Kondo fixed point, the conventional NRG method fails and its further developed version, the density matrix NRG (DM-NRG) $\stackrel{41}{\underline{1}}$ needs to be applied. Furthermore, a rather large number of multiplets must be kept to achieve good accuracy. We have therefore implemented a modified version of the recently developed spectral sum conserving DM-NRG method, where we use non-Abelian symmetries in a flexible way to compute the real and the imaginary parts of various local correlation functions $\underline{\underline{42}}$

To identify the relevant perturbations around the NFL fixed point we apply the machinery of boundary conformal field theory. Then we systematically study how the vicinity of fixed points and the introduction of relevant perturbations such as a finite channel anisotropy or a finite magnetic field influence the form of the dynamical response functions at zero temperature. We mainly focus on the strong coupling regime of the $2 \mathrm{CK}$ model and the universal cross-over functions in the proximity of this region induced by an external magnetic field or channel anisotropy. We remark that these cross-over functions, describing the cross-over from the non-Fermi liquid fixed point to a Fermi liquid fixed point, as well as the response functions can currently be computed reliably at all energy scales only with NRG. However, we shall be able to use the results of boundary conformal field theory, more precisely, the knowledge of the operator content of the two-channel Kondo fixed point and the scaling dimensions of the various perturbations around it, to make very general statements on the analytic properties of the various cross-over and spectral functions.

We shall devote special attention to superconducting fluctuations. It has been proposed that unusual superconducting states observed in some incoherent heavy fermion compounds could also emerge as a result of local superconducting correlations associated with twochannel Kondo physics. ${ }^{5,43,44}$ Here we investigate some possible superconducting order parameters consistent with the conformal field theoretical predictions, and find that the dominant instability emerges in the so-called composite superconducting channel, as it was proposed by Coleman et al. $\underline{\underline{44}}$

The paper is organized as follows. In Section $\Pi$ starting from the one-dimensional, continuum formulation of the 2CKM we connect it to a dimensionless approximation of it suited to our DM-NRG calculations. We also provide the symmetry generators used in the conformal field theoretical and DM-NRG calculations. In Section III we use boundary conformal field theory to classify the boundary highest-weight fields of the electron-hole symmetrical 2CKM by their quantum numbers and identify the relevant perturbations around the $2 \mathrm{CK}$ fixed point. Based on this classification the fields are then expanded in leading order in terms of the operators of the free theory. In Section IV we describe the technical details of our DM-NRG calculations. In Sections V VI and VII we study the real and the imaginary parts of the retarded Green's functions of the local fermions, the impurity spin and the local superconducting order parameters. In each of these sections we first discuss the analytic forms of the susceptibilities in the asymptotic regions of the twochannel and single channel Kondo scaling regimes, as they follow from scaling arguments. Then we confirm our predictions by demonstrating how the expected corrections due to the relevant perturbations and the leading irrelevant operator present themselves in the DM-NRG data. Furthermore we determine the boundaries of the 2CK scaling regimes and derive universal scaling curves connecting the FL and NFL fixed points for each operator under study. Finally, our conclusions are drawn in Section VIII.

\section{HAMILTONIAN AND SYMMETRIES}

The two-channel Kondo model consists of an impurity with a magnetic moment $S=\frac{1}{2}$ embedded into a Fermi liquid (FL) of two types of electrons (labeled by the flavor or channel indices $\alpha=1,2$ ), and interacting with them through a simple exchange interaction,

$$
\begin{aligned}
\mathscr{H} & =\sum_{\alpha, \mu} \int_{-D_{F}}^{D_{F}} \mathrm{~d} k k c_{\alpha, \mu}^{\dagger}(k) c_{\alpha, \mu}(k) \\
& +\sum_{\alpha} \sum_{\mu, \nu} \frac{J_{\alpha}}{2} \int_{-D_{F}}^{D_{F}} \mathrm{~d} k \int_{-D_{F}}^{D_{F}} \mathrm{~d} k^{\prime} \vec{S} c_{\alpha \mu}^{\dagger}(k) \vec{\sigma}_{\mu \nu} c_{\alpha \nu}\left(k^{\prime}\right) .
\end{aligned}
$$

Here $c_{\alpha, \mu}^{\dagger}(k)$ creates an electron of flavor $\alpha$ in the $l=0$ angular momentum channel with spin $\mu$ and radial momentum $k$ measured from the Fermi momentum. In the Hamiltonian above we allowed for a channel anisotropy of the couplings, $J_{1} \neq J_{2}$, and denoted the Pauli matrices by $\vec{\sigma}$. In the first, kinetic term, we assumed a spherical Fermi surface and linearized the spectrum of the conduction electrons, $\xi(k) \approx v_{F} k=k$, but these assumptions are not crucial: Apart from irrelevant terms in the Hamiltonian, our considerations below carry over to essentially any local density of states with electron-hole symmetry. The fields $c_{\alpha, \mu}^{\dagger}(k)$ are normalized to satisfy the anticommutation relations

$$
\left\{c_{\alpha, \mu}^{\dagger}(k), c_{\beta, \nu}\left(k^{\prime}\right)\right\}=\delta_{\alpha, \beta} \delta_{\mu, \nu} \delta\left(k-k^{\prime}\right)
$$


and therefore the couplings $J_{\alpha}$ are just the dimensionless couplings, usually defined in the literature. Since we are interested in the low-energy properties of the system, an energy cut-off $D_{F}$ is introduced for the kinetic and the interaction energies. In heavy fermion systems, this large energy scale is in the range of the Fermi energy, $D_{F} \sim$ $E_{F}$, while for quantum dots, it is of the order of the single particle level spacing of the dot, $\delta \epsilon$ or its charging energy, $E_{C}$, whichever is smaller.

The Hamiltonian above possesses various symmetries. To see it, it is worth to introduce the left-moving fermion fields,

$$
\psi_{\alpha, \mu}(x) \equiv \int_{-D_{F}}^{D_{F}} d k e^{-i k x} c_{\alpha, \mu}(k),
$$

and to rewrite the Hamiltonian as

$$
\begin{aligned}
\mathscr{H}=\sum_{\alpha, \mu} \int \frac{\mathrm{d} x}{2 \pi} \psi_{\alpha, \mu}^{\dagger}(x) i \partial_{x} \psi_{\alpha, \mu}(x) & \\
& +\sum_{\alpha} \frac{J_{\alpha}}{2} \vec{S} \psi^{\dagger}(0) \vec{\sigma} \psi(0) .
\end{aligned}
$$

Then the total spin operators $\mathscr{J}^{i}$ defined as

$$
\begin{aligned}
\mathscr{J}^{i} & \equiv S^{i}+\int \frac{\mathrm{d} x}{2 \pi} J^{i}(x), \\
J^{i}(x) & \equiv \frac{1}{2} \sum_{\alpha}: \psi_{\alpha}^{\dagger}(x) \sigma^{i} \psi_{\alpha}(x):
\end{aligned}
$$

commute with the Hamiltonian and satisfy the standard $\mathrm{SU}(2)$ algebra,

$$
\left[\mathscr{J}^{i}, \mathscr{J}^{j}\right]=i \epsilon^{i j k} \mathscr{J}^{k}
$$

In the previous equations we suppressed spin indices and introduced the normal ordering : ... : with respect to the non-interacting Fermi sea. In a similar way we can define the "charge spin" density operators, for the channels $\alpha=$ 1,2 as

$$
\begin{aligned}
C_{\alpha}^{z}(x) & \equiv \frac{1}{2}: \psi_{\alpha}^{\dagger}(x) \psi_{\alpha}(x): \\
C_{\alpha}^{-}(x) & \equiv \psi_{\alpha \uparrow}(x) \psi_{\alpha \downarrow}(x), \quad C_{\alpha}^{+}(x) \equiv \psi_{\alpha \downarrow}^{\dagger}(x) \psi_{\alpha \uparrow}^{\dagger}(x) \\
C_{\alpha}^{ \pm}(x) & \equiv C_{\alpha}^{x}(x) \pm i C_{\alpha}^{y}(x)
\end{aligned}
$$

and the corresponding symmetry generators

$$
\mathscr{C}_{\alpha}^{i} \equiv \int \frac{\mathrm{d} x}{2 \pi} C_{\alpha}^{i}(x) \quad(i=x, y, z) .
$$

The generators $\mathscr{C}_{\alpha}^{i}$, which are related to the electron-hole symmetry, ${ }^{45}$ satisfy the same $\mathrm{SU}(2)$ algebra as the $\mathscr{J}^{i}$-s,

$$
\left[\mathscr{C}_{\alpha}^{i}, \mathscr{C}_{\beta}^{j}\right]=i \delta_{\alpha \beta} \epsilon^{i j k} \mathscr{C}_{\beta}^{k}
$$

and they also commute with the Hamiltonian, Eq. (4). Thus the Hamiltonian $\mathscr{H}$ has a symmetry $\operatorname{SU}_{C 1}(2) \times$
$\mathrm{SU}_{C 2}(2) \times \mathrm{SU}_{S}(2)$ in the charge and spin sectors for arbitrary couplings, $J_{\alpha}$.

To perform NRG calculations, we use the following approximation of the dimensionless Hamiltonian, ${ }^{29}$

$$
\begin{aligned}
\frac{2 \mathscr{H}}{D_{F}\left(1+\Lambda^{-1}\right)} & \approx \sum_{\alpha} \sum_{\mu, \nu} \frac{\tilde{J}_{\alpha}}{2} \vec{S} f_{0, \alpha, \mu}^{\dagger} \vec{\sigma}_{\mu \nu} f_{0, \alpha, \mu} \\
+ & \sum_{n=0}^{\infty} \sum_{\alpha, \mu, \nu} t_{n}\left(f_{n, \alpha, \mu}^{\dagger} f_{n+1, \alpha, \mu}+\text { h.c. }\right)
\end{aligned}
$$

with $\Lambda$ a discretization parameter and $\tilde{J}_{\alpha}=4 J_{\alpha} /(1+$ $\left.\Lambda^{-1}\right)$. The operator, $f_{0}$ creates an electron right at the impurity site, and can be expressed as

$$
f_{0, \alpha, \mu}=\frac{1}{\sqrt{2 D_{F}}} \int_{-D_{F}}^{D_{F}} d k c_{\alpha, \mu}(k) .
$$

The Hamiltonian Eq. (11) is also called the Wilson chain: it describes electrons hopping along a semi-infinite chain with a hopping amplitude $t_{n} \sim \Lambda^{-n / 2}$, and interacting with the impurity only at site 0 . In the NRG procedure, this Hamiltonian is diagonalized iteratively, and its spectrum is used to compute the spectral functions of the various operators. 29

We remark that the Wilson Hamiltonian is not identical to $\mathscr{H}$, since some terms are neglected along its derivation $^{29}$ Nevertheless, similar to $\mathscr{H}$, the Wilson Hamiltonian also possesses the symmetry $\operatorname{SU}_{C 1}(2) \times$ $\mathrm{SU}_{C 2}(2) \times \mathrm{SU}_{S}(2)$ for arbitrary $J_{1}$ and $J_{2}$ couplings 45 The corresponding symmetry generators have been enumerated in Table【. We can then use these symmetries to label every multiplet in the Hilbert space and every operator multiplet by the eigenvalues $\vec{J}^{2}=j(j+1)$ and $\overrightarrow{\mathscr{C}}_{\alpha}^{2}=c_{\alpha}\left(c_{\alpha}+1\right)$. Throughout this paper, we shall use these quantum numbers to classify states and operators.

In the presence of a magnetic field, i.e., when a term ${ }^{50}$

$$
H_{\text {magn }}=-g \mu_{B} B S^{z}
$$

is added to $\mathscr{H}$, the symmetry of the system breaks down to $\mathrm{SU}_{C 1}(2) \times \mathrm{SU}_{C 2}(2) \times \mathrm{U}_{S}(1)$, with the symmetry $\mathrm{U}_{S}(1)$ corresponding to the conservation of the $z$-component of the spin, $\mathscr{J}^{z}$ (see Table II). In the rest of the papers we shall use units where we set $g \mu_{B} \equiv 1$.

\section{THE NON-FERMI LIQUID FIXED POINT AND ITS OPERATOR CONTENT}

For $J_{1}=J_{2}=J$ and in the absence of an external magnetic field, the Hamiltonian, $\mathscr{H}$ possesses a dynamically generated energy scale, the so-called Kondo temperature,

$$
T_{K} \approx D_{F} e^{-1 / J}
$$

The definition of $T_{K}$ is somewhat arbitrary. In this paper, $T_{K}$ shall be defined as the energy $\omega$ at which for $J_{1}=J_{2}$ 


\begin{tabular}{cc}
\hline \hline Symmetry group & Generators \\
$\mathrm{SU}_{C \alpha}(2)$ & $\mathrm{C}_{\alpha}^{+}=\sum_{n=0}^{\infty}(-1)^{n} f_{n, \alpha, \uparrow}^{\dagger} f_{n, \alpha, \downarrow}^{\dagger}, \mathrm{C}_{\alpha}^{z}=\frac{1}{2} \sum_{n=0}^{\infty} \sum_{\mu}\left(f_{n, \alpha, \mu}^{\dagger} f_{n, \alpha, \mu}-1\right), \mathrm{C}_{\alpha}^{-}=\mathrm{C}_{\alpha}^{+\dagger}$ \\
$\mathrm{SU}_{S}(2)$ & $\overrightarrow{\mathrm{J}}=\vec{S}+\frac{1}{2} \sum_{n=0 \alpha, \mu, \nu}^{\infty} \sum_{n, \alpha, \mu} \vec{\sigma}_{\mu \nu} f_{n, \alpha, \nu}$ \\
\hline \hline
\end{tabular}

TABLE I: Generators of the used symmetries for the two-channel Kondo model computations. Sites along the Wilson chain are labeled by $n$ whereas $\alpha$ and $\mu, \nu$ are the channel and spin indices, respectively.

the spectral function of the composite fermion drops to half of its value assumed at $\omega=0$ (for further details see the end of this Section and Fig. (1). For $B=0$ and $J_{1}=J_{2}$, below this energy scale the physics is governed by the so-called two-channel Kondo fixed point.

The physics of the two-channel Kondo fixed point and its vicinity can be captured using conformal field theory. The two-channel Kondo finite size spectrum and its operator content has first been obtained using boundary conformal field theory by Affleck and Ludwig. 28 However, instead of charge SU(2) symmetries, Affleck and Ludwig used flavor $\mathrm{SU}(2)$ and charge $\mathrm{U}(1)$ symmetries to obtain the fixed point spectrum. 28 The use of charge $\mathrm{SU}(2)$ symmetries, however, has a clear advantage over the flavor symmetry when it comes to performing NRG calculations: While the channel anisotropy violates the flavor symmetry, it does not violate the charge $\mathrm{SU}(2)$ symmetries. Therefore, even in the channel anisotropic case, we have three commuting $\mathrm{SU}(2)$ symmetries. If we switch on a local magnetic field, only the spin $\mathrm{SU}(2)$ symmetry is reduced to its $\mathrm{U}(1)$ subgroup. Using charge symmetries allows thus for much more precise calculations, and in fact, using them is absolutely necessary to obtain satisfactorily accurate spectral functions, especially in the presence of a magnetic field.

To understand the fixed point spectrum and the operator content of the 2CKM, let us outline the boundary conformal field theory in this $\mathrm{SU}_{C 1}(2) \times \mathrm{SU}_{C 2}(2) \times \mathrm{SU}_{S}(2)$ language. First, we remark that the spin density operators, $J^{i}(x)$ satisfy the $\mathrm{SU}(2)_{k=2}$ Kac-Moody algebra of level $k=2$,

$$
\begin{aligned}
{\left[J^{i}(x), J^{j}\left(x^{\prime}\right)\right] } & =\frac{k}{2} \delta^{i j} \delta^{\prime}\left(x-x^{\prime}\right) \\
& +i 2 \pi \delta\left(x-x^{\prime}\right) \epsilon^{i j k} J^{k}(x)
\end{aligned}
$$

while the charge density operators, $C_{\alpha}^{i}(x)$ defined in the previous section satisfy the Kac-Moody algebra of level $k=1$ :

$$
\begin{aligned}
{\left[C_{\alpha}^{i}(x), C_{\beta}^{j}\left(x^{\prime}\right)\right] } & =\frac{k}{2} \delta^{i j} \delta_{\alpha \beta} \delta^{\prime}\left(x-x^{\prime}\right) \\
& +i 2 \pi \delta_{\alpha \beta} \delta\left(x-x^{\prime}\right) \epsilon^{i j k} C_{\alpha}^{k}(x)
\end{aligned}
$$

We can use these current densities and the coset con- struction to write the kinetic part of the Hamiltonian as

$$
\begin{aligned}
\mathscr{H}_{0} & =\mathscr{H}_{C 1}+\mathscr{H}_{C 2}+\mathscr{H}_{S}+\mathscr{H}_{I}, \\
\mathscr{H}_{C \alpha} & =\frac{1}{3} \int \frac{\mathrm{d} x}{2 \pi}: \vec{C}_{\alpha}(x) \vec{C}_{\alpha}(x):, \\
\mathscr{H}_{S} & =\frac{1}{4} \int \frac{\mathrm{d} x}{2 \pi}: \vec{J}(x) \vec{J}(x): .
\end{aligned}
$$

In $\mathscr{H}_{0}$, the first two terms describe the charge sectors, and have central charge $c=1$, while $\mathscr{H}_{S}$ describes the spin sector, and has central charge $c=3 / 2$. The last term corresponds to the coset space, and must have central charge $c=1 / 2$, since the free fermion model has central charge $c=4$, corresponding to the four combinations of spin and channel quantum numbers. This term can thus be identified as the Ising model, having primary fields $\mathbb{1}, \sigma, \epsilon$ with scaling dimensions $0,1 / 16,1 / 2$, respectively. We can then carry out the conformal embedding in the usual way, by comparing the finite size spectrum of the free Hamiltonian with that of Eq. (15), and identifying the allowed primary fields in the product space. The the fusion rules obtained this way are listed on the left side of Table [II. The finite size spectrum at the twochannel Kondo fixed point can be derived by fusing with the impurity spin (which couples to the spin sector only), following the operator product expansion of the WessZumino-Novikov-Witten model, $1 / 2 \otimes 0 \rightarrow 1 / 2,1 / 2 \otimes$ $1 / 2 \rightarrow 0 \oplus 1,1 / 2 \otimes 1 \rightarrow 1 / 2$ (see RHS of TableЩ). Finally, the operator content of the fixed point can be found by performing a second fusion with the spin. The results of this double fusion are presented in Table【II In Table【I the leading irrelevant operator, $\overrightarrow{\mathcal{J}}_{-1} \vec{\phi}_{s}$, is also included. Although it is not a primary field, $\stackrel{28}{=}$ close to the $2 \mathrm{CK}$ fixed point, this operator will also have impact on the form the correlation functions.

What remains is to identify the scaling operators in terms of the operators of the non-interacting theory. In general, an operator of the non-interacting theory can be written as an infinite series in terms of the scaling operators and their descendants. Apart from the Ising sector, which is hard to identify, we can tell by looking at the various quantum numbers of an operator acting on the Wilson chain, which primary fields could be present in it. In this way, we can identify, e.g. $\vec{\phi}_{s}$ as the spin operator $\vec{S}$. Thus the spin operator can be expressed as

$$
\vec{S}=A_{s} \vec{\phi}_{s}+\ldots
$$




\begin{tabular}{cccc|c}
\hline \hline$c_{1}$ & $c_{2}$ & $j$ & $I$ & $E_{\text {free }}$ \\
\hline 0 & 0 & 0 & 1 & 0 \\
$\frac{1}{2}$ & 0 & $\frac{1}{2}$ & $\sigma$ & $\frac{1}{2}$ \\
0 & $\frac{1}{2}$ & $\frac{1}{2}$ & $\sigma$ & $\frac{1}{2}$ \\
$\frac{1}{2}$ & $\frac{1}{2}$ & 1 & 1 & 1 \\
$\frac{1}{2}$ & $\frac{1}{2}$ & 0 & $\epsilon$ & 1 \\
\hline \hline
\end{tabular}

\begin{tabular}{cccc|c}
\hline \hline$c_{1}$ & $c_{2}$ & $j$ & $I$ & $E_{2 \mathrm{CKM}}$ \\
\hline 0 & 0 & $\frac{1}{2}$ & 1 & 0 \\
$\frac{1}{2}$ & 0 & 0 & $\sigma$ & $\frac{1}{8}$ \\
0 & $\frac{1}{2}$ & 0 & $\sigma$ & $\frac{1}{8}$ \\
$\frac{1}{2}$ & $\frac{1}{2}$ & $\frac{1}{2}$ & 1 & $\frac{1}{2}$ \\
$\frac{1}{2}$ & 0 & 1 & $\sigma$ & $\frac{5}{8}$ \\
0 & $\frac{1}{2}$ & 1 & $\sigma$ & $\frac{5}{8}$ \\
$\frac{1}{2}$ & $\frac{1}{2}$ & $\frac{1}{2}$ & $\epsilon$ & 1 \\
\hline \hline
\end{tabular}

TABLE II: Left: Primary fields and the corresponding finite size energies at the free fermion fixed point for anti-periodic boundary conditions. States are classified according to the group $\mathrm{SU}_{C 1}(2) \times \mathrm{SU}_{C 2}(2) \times \mathrm{SU}_{S}(2)$ and the Ising model. The excitation energies $E_{\text {free }}$ are given in units of $2 \pi / L$, with $L$ the size of the chiral fermion system. Right: Finite size spectrum at the two-channel Kondo fixed point.

where the dots stand for all the less relevant operators that are present in the expansion of $\vec{S}$, and some highfrequency portions which are not properly captured in the expansion above. The weight, $A_{s}$, can be determined from matching the decay of the spin-spin correlation function at short and long times. This way we end up with $A_{s} \sim 1 / \sqrt{T_{K}}$.

We remark that there are infinitely many operators that contain the scaling fields in their expansion. As an example, consider the operators $\phi_{\psi 1}^{\tau \sigma}$. Here the label $\sigma=\{\uparrow, \downarrow\}$ refers to the spin components of a $j=1 / 2$ spinor, while $\tau= \pm$ refer to the charge spins of a charge $c=1 / 2$ spinor. To identify the corresponding operator on the Wilson chain, we first note that $f_{0,1, \sigma}^{\dagger}$ transforms as a spinor under spin rotations. It can easily be seen that the operator $\tilde{f}_{0,1}^{\dagger} \equiv i \sigma_{y} f_{0,1}$ also transforms as a spinor. We can then form a four-spinor out of these operators, $\gamma_{1} \equiv\left\{f_{0,1, \sigma}^{\dagger}, \tilde{f}_{0,1, \sigma}^{\dagger}\right\}$. It is easy to show that $\gamma_{1}$ transforms as a spinor under $\mathrm{SU}_{C 1}(2)$ rotations as well, thus $\phi_{\psi 1}^{\tau \sigma}$ could be identified as $\gamma_{1}=\left\{f_{0,1, \sigma}^{\dagger}, \tilde{f}_{0,1, \sigma}^{\dagger}\right\}$.

However, we can construct another operator, $F_{1}^{\dagger} \equiv$ $f_{0,1}^{\dagger} \vec{S} \vec{\sigma}$ and its counterpart, $\tilde{F}_{1}^{\dagger} \equiv i \sigma_{y} F_{1}$, and form a fourspinor out of them: $\Gamma_{1} \equiv\left\{F_{1, \sigma}^{\dagger}, \tilde{F}_{1, \sigma}^{\dagger}\right\}$. This operator has the same quantum numbers as $\gamma_{1}$, and in fact, both operators' expansion contains $\phi_{\psi 1}^{\tau \sigma}$.

The operator $\phi_{\Delta}^{\tau \tau^{\prime}}$ is of special interest, since it is relevant at the two-channel Kondo fixed point, just like the spin. Its susceptibility therefore diverges logarithmically. Good candidates for these operators would be $\sum_{\sigma \sigma^{\prime}} \epsilon_{\sigma \sigma^{\prime}} \gamma_{1}^{\tau \sigma} \gamma_{2}^{\tau^{\prime} \sigma^{\prime}}$, since these are spin singlet operators that behave as charge $1 / 2$ spinors in both channels. The $\tau=\tau^{\prime}=+$ component of this operator corresponds to the superconducting order parameter

$$
\mathcal{O}_{S C} \equiv f_{0,1, \uparrow}^{\dagger} f_{0,2, \downarrow}^{\dagger}-f_{0,1, \downarrow}^{\dagger} f_{0,2, \uparrow}^{\dagger},
$$

while the +- components describe simply a local operator that hybridizes the channels, $\sim f_{0,1, \sigma}^{\dagger} f_{0,2, \sigma}$.
Another candidate would be the operator, $\sum_{\sigma \sigma^{\prime}} \epsilon_{\sigma \sigma^{\prime}} \Gamma_{1}^{\tau \sigma} \gamma_{2}^{\tau^{\prime} \sigma^{\prime}}$. This operator is also a local singlet, and has charge spins $c_{1}=c_{2}=1 / 2$. It contains the following component of the composite superconducting order parameter

$$
\mathcal{O}_{S C C} \equiv f_{0,1}^{\dagger} \vec{S} \vec{\sigma} i \sigma_{y} f_{0,2}^{\dagger} .
$$

From their transformation properties it is not obvious, which one of the above superconducting order parameters gives the leading singularity. However, NRG gives a very solid answer and tells us that, while the susceptibility of the traditional operator does not diverge as the temperature or frequency goes to zero, that of the composite order parameter does. It is thus this latter operator that can be identified as $\phi_{\Delta}^{\tau \tau^{\prime}}$. Note that, in case of electron-hole symmetry, the composite hybridization operator

$$
\mathcal{O}_{\text {mix }} \equiv f_{0,1}^{\dagger} \vec{S} \vec{\sigma} f_{0,2}
$$

has the same singular susceptibility as $\mathcal{O}_{S C C}$ since they are both components of the same tensor operator. This is, however, not true any more away from electron-hole symmetry. Furthermore, superconducting correlations are usually more dangerous, since in the Cooper channel any small attraction would lead to ordering when a regular lattice model of two-channel Kondo impurities is considered.

The knowledge of the operator content of the twochannel Kondo fixed point enables us to describe the effects of small magnetic fields and small channel anisotropies $\left(J_{1} \neq J_{2}\right)$. For energies and temperatures below $T_{K}$, the behavior of the model can be described by the slightly perturbed two-channel Kondo fixed point Hamiltonian. For $J_{1} \approx J_{2}$ and in a small magnetic field, $B \ll T_{K}$, this Hamiltonian can be expressed as

$$
\begin{aligned}
& \mathcal{H}=\mathcal{H}_{2 C K}^{*}+ \\
& +D_{0}^{1 / 2} \kappa_{0} \phi_{\text {anis }}+D_{0}^{1 / 2} \vec{h}_{0} \vec{\phi}_{s}+D_{0}^{-1 / 2} \lambda_{0} \overrightarrow{\mathcal{J}}_{-1} \vec{\phi}_{s}+\ldots
\end{aligned}
$$

Here $\mathcal{H}_{2 C K}^{*}$ is the $2 \mathrm{CK}$ fixed point Hamiltonian, and $\kappa_{0}$ is the dimensionless coupling to the channel anisotropy field, $\phi_{\text {anis }}$, whereas the effective magnetic field, $\vec{h}_{0}$, couples to the "spin field", $\phi_{s}$. Both of them are relevant perturbations at the two-channel Kondo fixed point and they must vanish to end up with the two-channel Kondo fixed point at $\omega, T \rightarrow 0$. The third coupling, $\lambda_{0}$, couples to the leading irrelevant operator (see Tab. III), which dominates the physics when $\kappa=h=0$. The energy cutoff $D_{0}$ in Eq. (20) is a somewhat arbitrary scale: it can be though of as the energy scale below which the twochannel Kondo physics emerges, i.e. $D_{0} \sim T_{K}$. Then the dimensionless couplings $\kappa_{0}, \lambda_{0}$ and $h_{0}$ are approximately related to the couplings of the original Hamilto- 


\begin{tabular}{|c|c|c|c|c|c|c|}
\hline$\overline{c_{1}}$ & $\overline{c_{2}}$ & $\bar{j}$ & $\overline{I 2}$ & $\overline{x^{2 C K}}$ & $\begin{array}{c}\text { scaling } \\
\text { operators }\end{array}$ & corresponding operators \\
\hline$\overline{0}$ & 0 & 1 & 1 & $\frac{1}{2}$ & $\vec{\phi}_{s}$ & $\vec{S}$ \\
\hline$\frac{1}{2}$ & 0 & $\underline{1}$ & & $\frac{1}{2}$ & $\phi_{\psi 1}^{\tau \sigma}$ & $\begin{array}{c}\gamma_{1} \equiv\left(f_{0,1, \sigma}^{\dagger},\left(i \sigma_{y} f_{0,1}\right)_{\sigma}\right) \\
\Gamma_{1} \equiv\left(F_{0,1, \sigma}^{\dagger},\left(i \sigma_{y} F_{0,1}\right)_{\sigma}\right)\end{array}$ \\
\hline 0 & $\frac{1}{2}$ & $\frac{1}{2}$ & & $\frac{1}{2}$ & $\phi_{\psi 2}^{\tau \sigma}$ & 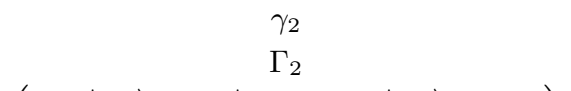 \\
\hline$\frac{1}{2}$ & $\frac{1}{2}$ & 0 & & $\frac{1}{2}$ & $\phi_{\Delta}^{\tau \tau^{\prime}}$ & $\left(\begin{array}{cc}f_{0,1}^{\dagger} \vec{S} \vec{\sigma} i \sigma_{y} f_{0,2}^{\dagger} & -f_{0,1}^{\dagger} \vec{S} \vec{\sigma} f_{0,2} \\
-f_{0,1} \sigma_{y} \vec{S} \vec{\sigma} \sigma_{y} f_{0,2}^{\dagger} & -f_{0,1} i \sigma_{y} \vec{S} \vec{\sigma} f_{0,2}\end{array}\right)$ \\
\hline 0 & 0 & 0 & & $\frac{1}{2}$ & $\begin{array}{l}\phi_{\text {anis }} \\
\overrightarrow{\vec{\tau}} \overrightarrow{\vec{s}}\end{array}$ & $\vec{S}\left(f_{0,1}^{\dagger} \vec{\sigma} f_{0,1}-f_{0,2}^{\dagger} \vec{\sigma} f_{0,2}\right)$ \\
\hline 0 & 0 & 01 & & $\frac{3}{2}$ & $\overline{\mathcal{J}}_{-1} \vec{\phi}_{s}$ & $\vec{S}\left(f_{0,1}^{\dagger} \vec{\sigma} f_{0,1}+f_{0,2}^{\dagger} \vec{\sigma} f_{0,2}\right)$ \\
\hline
\end{tabular}

TABLE III: Highest-weight operators and their dimensions $x^{2 C K}$ at the $2 \mathrm{CK}$ fixed point. Operators are classified by the symmetry group $\mathrm{SU}_{C 1}(2) \times \mathrm{SU}_{C 2}(2) \times \mathrm{SU}_{S}(2)$ and the scaling operators of the Ising model. The constants $c_{1}$ and $c_{2}$ denote the charge spins in channels 1 and 2, respectively, while $j$ refers to the spin, and $I$ labels the scaling operators of the Ising model: $\mathbb{1 1}, \sigma, \epsilon$. Superscripts $\tau, \tau^{\prime}= \pm$ refer to the two components of charge spinors, while $\sigma=\uparrow, \downarrow$ label the components of a spin- $\pm \frac{1}{2}$ spinor.

nian, Eq. (11), as

$$
\begin{aligned}
\kappa_{0} & \approx K_{R} \equiv 4 \frac{J_{1}-J_{2}}{\left(J_{1}+J_{2}\right)^{2}}, \\
h_{0} & \approx B / T_{K}, \\
\lambda_{0} & \approx O(1) .
\end{aligned}
$$

However, the arbitrary scale $D_{0}$ in Eq. (20) can be changed at the expense of changing the couplings: $D_{0} \rightarrow$ $D, \kappa_{0} \rightarrow \kappa(D), h_{0} \rightarrow h(D)$ and $\lambda_{0} \rightarrow \lambda(D)$ in such a way that the physics below $D_{0}$ remains unchanged. This freedom translates to scaling equations, whose leading terms follow from the conformal field theory results, and read

$$
\begin{aligned}
& \frac{d \kappa(D)}{d x}=\frac{1}{2} \kappa(D)+\ldots, \\
& \frac{d h(D)}{d x}=\frac{1}{2} h(D)+\ldots, \\
& \frac{d \lambda(D)}{d x}=-\frac{1}{2} \lambda(D)+\ldots,
\end{aligned}
$$

with $x=-\log D$. Solving these equations with the initial conditions, $D=D_{0} \sim T_{K}$ and $h=h_{0}, \kappa=\kappa_{0}, \lambda=\lambda_{0}$, we can read out the energy scales at which the rescaled couplings become of the order of one,

$$
\begin{aligned}
& T^{*} \propto T_{K} \kappa_{0}^{2} \sim T_{K} \frac{\left(J_{1}-J_{2}\right)^{2}}{\left(J_{1}+J_{2}\right)^{4}}, \\
& T_{h} \propto T_{K} h_{0}^{2} \sim B^{2} / T_{K} .
\end{aligned}
$$

At these scales the couplings of the relevant operators are so large that they can no longer be treated as perturbations. Below $T^{*}$ the single channel Kondo behavior is recovered in the more strongly coupled channel, while $T_{h}$ can be interpreted as the scale where the impurity spin dynamics is frozen by the external field.
The prefactors in Eqs. (28) are somewhat arbitrary, and depend slightly on the precise definition one uses to extract these scales. In this paper, we shall use the spectral function of the composite fermion to define the scales $T_{K}$ and $T^{*}$. We define $T_{K}$ to be the energy at which for $K_{R}=0$ the spectral function of the composite fermion takes half of its fixed point value (i.e. the value assumed at $\omega=0)$. Whereas $T^{*}$ is the energy at which for $K_{R}>0$ it takes $75 \%$ of its fixed point value (see Fig. (1).

It is much harder to relate $T_{h}$ to a physically measurable quantity. We defined it simply through the relation,

$$
T_{h} \equiv C_{h} \frac{B^{2}}{T_{K}},
$$

where the constant was chosen to be $C_{h} \approx 60$. This way $T_{h}$ corresponds roughly to the energy at which the NFL finite size spectrum crosses over to the low-frequency FL spectrum.

\section{NRG CALCULATIONS}

Prior to discussing the analytic and numerical features of the response functions, let us devote this section to the short description of the NRG procedure used. All results presented in this paper refer to zero temperature. The NRG calculations were performed with a discretization parameter $\Lambda=2$. The sum of the dimensionless couplings was $\tilde{J}_{1}+\tilde{J}_{2}=0.4$ for each run. The NRG data were computed with a so-called flexible DM-NRG program,, 42 which permits the use of an arbitrary number of Abelian and non-Abelian symmetries (see Tab. I), and incorporates the spectral-sum conserving density matrix NRG

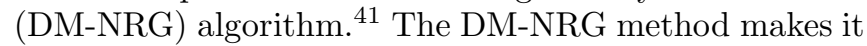
possible to generate spectral functions that satisfy spectral sum rules with machine precision at $T=0$ tem- 


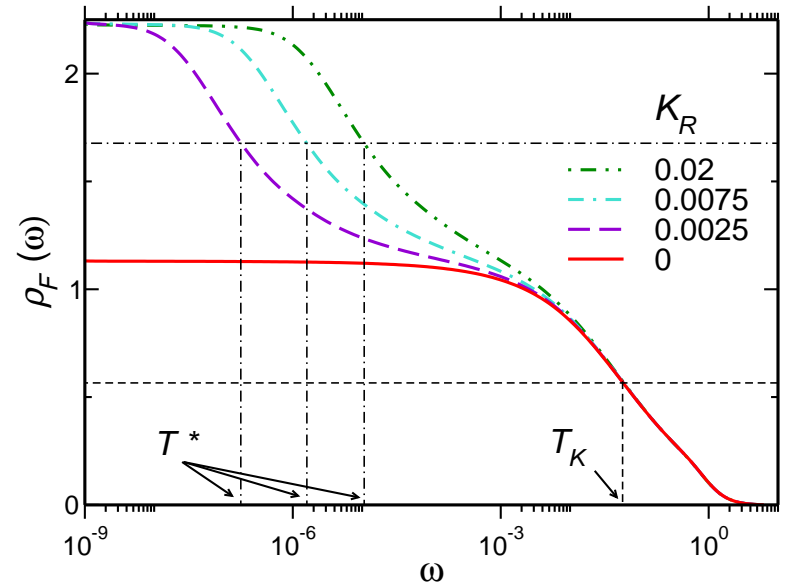

FIG. 1: (color online) Spectral function $\varrho_{F}$ of the composite fermion operator, $F_{0,1, \uparrow}$ as a function of $\omega$, and the definition of the scales $T_{K}$ and $T^{*}$. $T_{K}$ is defined by the relation $\varrho_{F}(\omega=$ $\left.T_{K}, T=0, K_{R}=0\right) \equiv \frac{1}{2} \varrho_{F}\left(\omega=0, T=0, K_{R}=0\right)$. For nonzero $K_{R}$ the scale $T^{*}$ is defined through $\varrho_{F}\left(\omega=T^{*}, T=\right.$ $\left.0, K_{R}\right) \equiv \frac{3}{4} \varrho_{F}\left(\omega=0, T=0,\left|K_{R}\right|\right)$.

perature. For calculations with non-zero magnetic field the use of the DM-NRG method represents a great advantage over conventional NRG methods, $\stackrel{46}{,}$ which loose spectral weights and violate spectral sum rules. Conventional methods also lead to smaller or bigger jumps in the spectral functions at $\omega=0$ which hinder the computation of the universal scaling functions provided by the scale $T_{h}{ }^{24}$ The DM-NRG method solves all these problems if a sufficient number of multiplets is kept. On an ordinary desk-top computer, however, we need to use as many symmetries as possible to keep the computation time within reasonable limits.

In the present paper, where we study the electronhole symmetrical case, it is possible to use the symmetry group $\mathrm{SU}_{C 1}(2) \times \mathrm{SU}_{C 2}(2) \times \mathrm{SU}_{S}(2)$ even in case of channel anisotropy. At these calculations the maximum number of kept multiplets was 750 in each iteration. This corresponds to the diagonalization of $\approx 85$ matrices with matrix sizes ranging up to $\approx 630$, acting on the vector space of $\approx 9000$ multiplets consisting of $\approx 106000$ states. In the presence of magnetic field we used the symmetry group $\mathrm{SU}_{C 1}(2) \times \mathrm{SU}_{C 2}(2) \times \mathrm{U}_{S}(1)$, and retained a maximum of 1350 multiplets in each iteration, that corresponds to the diagonalization of $\approx 150$ matrices with matrix sizes ranging up to $\approx 800$ acting on the vector space of $\approx 18000$ multiplets consisting of $\approx 73000$ states.

In the next sections, we shall see how the knowledge of the operator content of the two-channel Kondo fixed point can help us to understand the analytic structure of the various dynamical correlation functions obtained by NRG.

\section{LOCAL FERMIONS' SPECTRAL FUNCTIONS AND SUSCEPTIBILITIES}

Let us first analyse the Green's function of the local fermion, $f_{0, \sigma, \alpha}^{\dagger} \leftrightarrow \vec{\gamma}_{\alpha}$. The composite fermion's $\left(F_{0, \sigma, \alpha}^{\dagger} \leftrightarrow\right.$ $\left.\vec{\Gamma}_{0, \alpha}\right)$ Green's function has already been looked into in detail in an earlier study of ours ${ }^{24}$ We shall therefore not discuss its analytic properties here but use it merely as a reference to define the various energy scales in the NRG calculations (see Fig. 1). Let us note, however, that in the large bandwidth limit, $\omega, T_{K} \ll D_{F}$, the spectral function of the composite fermion and that of the local fermion are simply related,

$$
\varrho_{f}(\omega)=\frac{1}{2 D_{F}}-\frac{\pi}{4} J^{2} \varrho_{F}(\omega) .
$$

Thus, apart from a trivial constant shift and a minus sign, the spectral function of the local fermion is that of the composite fermion, and all features of $\varrho_{F}$ are also reflected in $\varrho_{f}$.

Before we discuss the NRG results, let us examine what predictions we have for the retarded Green's function of the operator $f_{0, \sigma, \alpha}^{\dagger}$ from conformal field theory. By looking at its quantum numbers, this operator can be identified with the operator $\phi_{\psi \alpha}^{+\sigma}$ (see Tab. III), i.e.

$$
f_{0, \sigma, \alpha}^{\dagger}=A_{f} \phi_{\psi \alpha}^{+\sigma}+\ldots
$$

with the prefactor $A_{f} \propto 1 / \sqrt{D_{F}}$. Note that $A_{f}$ is a complex number, it does not need to be real. The dots in the equation above indicate the series of other, less relevant operators and their descendants, which give subleading corrections to the correlation function of $f_{0, \sigma, \alpha}^{\dagger}$. Furthermore, the expansion above holds for the long time behavior. The "short time part" of the correlation function of $f_{0, \sigma, \alpha}^{\dagger}$ is not captured by Eq. (31), and gives a constant to $\mathcal{G}_{f}(\omega)$ of the order of $\sim 1 / D_{F}$. Thus, apart from a prefactor $A_{f}^{2}$, a constant shift and subleading terms, the Green's function of $f_{0, \sigma, \alpha}^{\dagger}$ is that of the field $\phi_{\psi \alpha}^{+\sigma}$. As we discuss it shortly in Appendix $\mathrm{A}$, the Fourier transform of the Green's function of any operator of dimension $x=1 / 2$ is scale invariant around the two-channel Kondo fixed point. Since $\phi_{\psi \alpha}^{+\sigma}$ and thus $f_{0, \sigma, \alpha}^{\dagger}$ have a scaling dimension $1 / 2$ at the $2 \mathrm{CK}$ fixed point, it follows that the dimensionless retarded Green's function, $D_{F} \mathcal{G}_{f}(\omega)$, is also scale invariant, 51

$$
\begin{aligned}
D_{F} \mathcal{G}_{f}(\omega, T) & \equiv \hat{g}_{f}\left(\frac{\omega}{D}, \frac{T}{D}, \kappa(D), h(D), \lambda(D), \ldots\right), \\
D \frac{\mathrm{d} \hat{g}_{f}}{\mathrm{~d} D} & =0 .
\end{aligned}
$$

From Eq. (32), we can deduce various important properties. Let us first consider the simplest case, $T=0$ and $\kappa=h=0$. Then setting the scale $D$ to $D_{0} \sim T_{K}$ we have

$$
\hat{g}_{f}^{\kappa, h, T=0}(\omega)=\hat{g}_{f}\left(\frac{\omega}{D_{0}}, \lambda_{0}, \ldots\right) .
$$


Let us now rescale $D \rightarrow|\omega|$, and use the fixed point scaling equation (26) to obtain $\lambda(D)$,

$$
\hat{g}_{f}=g_{f}\left( \pm 1, \sqrt{\frac{|\omega|}{D_{0}}} \lambda_{0}\right) .
$$

Assuming that this function is analytic in its second argument we obtain for $|\omega| \ll T_{K}$

$$
\begin{aligned}
\hat{g}_{f}^{\kappa, h, T=0}(\omega) & =\hat{g}_{f}\left(\frac{\omega}{T_{K}}\right) \\
& \approx g_{ \pm f}+g_{ \pm f}^{\prime} \sqrt{\frac{|\omega|}{T_{K}}}+\ldots,
\end{aligned}
$$

with $g_{ \pm f}$ and $g_{ \pm f}^{\prime}$ some complex expansion coefficients. Here the subscripts \pm refer to the cases $\omega>0$ and $\omega<0$, respectively. As we discussed above, the constants $g_{ \pm} f$ depend also on the short time behavior of $\mathcal{G}_{f}(t)$, and are not universal in this sense. These constants are not independent of each other. They are related by the constraint that the Green's function must be analytic in the upper half-plane. Furthermore, electron-hole symmetry implies that $g_{+f}=g_{-f}$ and $g_{+f}^{\prime}=-\left(g_{-f}^{\prime}\right)^{*}$.

Relations similar to the ones above hold for the dimensionless spectral function. It is defined as

$$
\hat{\varrho}_{f}(\omega) \equiv-\frac{1}{\pi} \operatorname{Im} \hat{g}_{f}(\omega),
$$

and assumes the following simpler form at small frequencies in case of electron-hole symmetry,

$$
\hat{\varrho}_{f}^{T, \kappa, h=0}(\omega)=r_{f}+r_{f}^{\prime} \sqrt{\frac{|\omega|}{T_{K}}}+\ldots
$$

For $\omega \gg T_{K}$ the scaling dimension of the local fermion is $x_{f}^{\text {free }}=1 / 2$ corresponding to an $\omega$-independent spectral function. Perturbation theory in $J$ amounts to logarithmic corrections of the form: $1 / 2-c s t / \log ^{2}\left(T_{K} / \omega\right)$, as it is sketched in the upper parts of Fig.-s 2 and 3 .

For $T \neq 0$, and $\kappa=h=0$ using similar arguments as before, but now rescaling $D \rightarrow T$ we find

$$
\begin{aligned}
\hat{g}_{f}^{\kappa, h=0}(\omega) & =\hat{g}_{f}\left(\frac{\omega}{T}, \frac{T}{T_{K}}, \lambda_{0}\right) \\
& \equiv \hat{g}_{f}\left(\frac{\omega}{T}, 1, \sqrt{\frac{T}{D_{0}}} \lambda_{0}, \ldots\right) .
\end{aligned}
$$

Then by expanding $\hat{g}_{f}$ we obtain the following scaling form for the low temperature behavior of the spectral function,

$$
\hat{\varrho}_{f}^{h, \kappa=0}(\omega)=\Theta_{f}\left(\frac{\omega}{T}\right)+\sqrt{\frac{T}{T_{K}}} \tilde{\Theta}_{f}\left(\frac{\omega}{T}\right)+\ldots,
$$

with $\Theta_{f}$ and $\tilde{\Theta}_{f}$ universal scaling functions. Note that we made no assumption on the ratio $\omega / T$, but both $\omega$ and
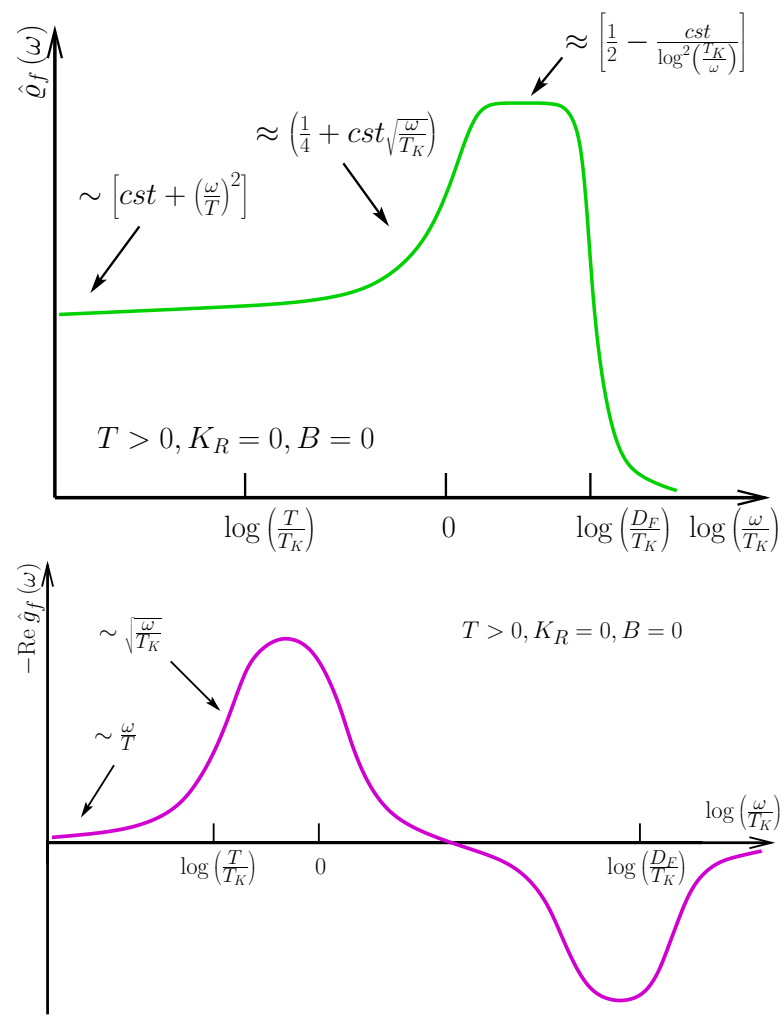

FIG. 2: (color online) (top) Sketch of the dimensionless spectral function $\hat{\varrho}_{f}=D_{F} \varrho_{f}$ of $f_{0,1, \sigma}^{\dagger}$, and (bottom) the real part of its dimensionless Green's function, Re $\hat{g}_{f}=D_{F} \operatorname{Re} \mathcal{G}_{f}$ for $T>0$ and $K_{R}=0, B=0$ as a function of $\log \left(\omega / T_{K}\right)$. Asymptotics indicated for $\omega<T_{K}$ were derived through scaling arguments. The large $\omega$-behavior is a result of perturbation theory.

$T$ must be smaller than $T_{K}$. The asymptotic properties of $\Theta_{f}$ and $\tilde{\Theta}_{f}$ can be extracted by making use of the facts that $(i) \hat{g}_{f}(\omega, T)$ must be analytic for $\omega \ll T$, $(i i)$ that Eq. (39) should reproduce the $T \rightarrow 0$ results in the limit $\omega \gg T$, and (iii) that by electron-hole symmetry, $\hat{\varrho}_{f}$ must be an even function of $\omega$. The issuing asymptotic properties together with those of the other scaling functions defined later are summarized in Table $\mathrm{V}$. The asymptotic properties of the real part, Re $\hat{g}_{f}$, can be extracted from those of $\hat{\varrho}_{f}$ by performing a Hilbert transform

$$
\operatorname{Re} \hat{g}_{f}(\omega)=\mathcal{P} \int \mathrm{d} \tilde{\omega} \frac{\hat{\varrho}_{f}(\tilde{\omega})}{\omega-\tilde{\omega}}
$$

with $\mathcal{P}$ the principal part. The obtained features are sketched in Fig. 2 for $T>0$ and $\kappa=h=0$.

Let us now investigate the effect of channel anisotropy, i.e. $\kappa \neq 0$ at $T=0$ temperature and no magnetic field $h=0$. In this case, we can rescale $D$ to $D=|\omega|$ to obtain

$$
\hat{\varrho}_{f}^{T, h=0}(\omega)=\mathcal{K}_{f}^{ \pm}\left(\frac{\omega}{T^{*}}\right)+\sqrt{\frac{|\omega|}{T_{K}}} \tilde{\mathcal{K}}_{f}^{ \pm}\left(\frac{\omega}{T^{*}}\right)+\ldots,
$$




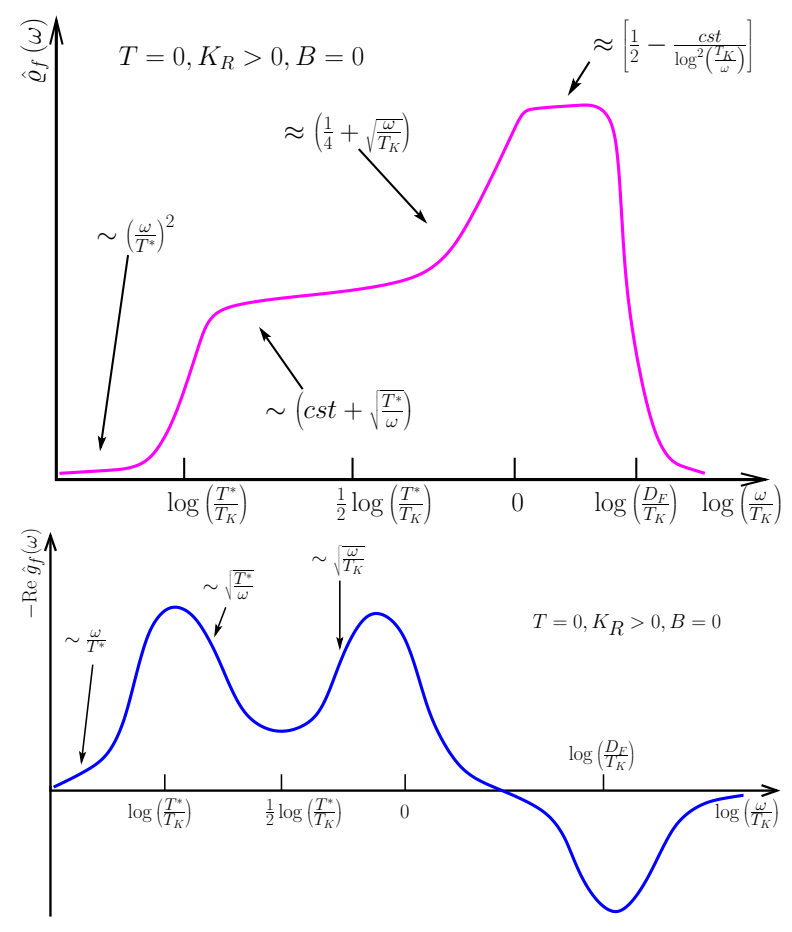

FIG. 3: (color online) (top) Sketch of the dimensionless spectral function of $f_{0,1, \sigma}^{\dagger}: \hat{\varrho}_{f}=D_{F} \varrho_{f}$, and (bottom) the real part of its dimensionless Green's function: $\operatorname{Re} \hat{g}_{f}=D_{F} \operatorname{Re} \mathcal{G}_{f}$ for $T=0, K_{R}>0$ and $B=0$ as a function of $\log \left(\omega / T_{K}\right)$. Asymptotics indicated for $\omega<T_{K}$ were derived through scaling arguments. The large $\omega$-behavior is a result of perturbation theory.

with $T^{*}$ the anisotropy scale defined earlier. The superscripts \pm refer to the cases of positive or negative anisotropies: the superscript "+" is used when the coupling is larger in the channel where we measure the Green's function of $f_{0, \alpha, \sigma}^{\dagger}$. The asymptotics of the universal functions $\mathcal{K}_{f}^{ \pm}$and $\tilde{\mathcal{K}}_{f}^{ \pm}$can be obtained through similar scaling arguments as before and they differ only slightly from those of $\Theta_{f}$ and $\tilde{\Theta}_{f}$ (see Table $\square$ for a summary). The properties of $\hat{\varrho}_{f}^{T, h=0}(\omega)$ are summarized in Fig. 3 , A remarkable feature of the spectral function is that it contains a correction $\sim \sqrt{T^{*} /|\omega|}$. This correction can be obtained by doing perturbation theory in the small parameter $\kappa(\omega)$ at the two-channel Kondo fixed point.

From the asymptotic forms in Table $\mathrm{V}$ we find that in the local fermion's susceptibility a new scale, $T_{f}^{* *} \sim$ $\sqrt{T^{*} T_{K}}$ appears as a result of the competition between the leading irrelevant operator and the channel anisotropy ${ }^{24}$ It is only in the regime $T_{f}^{* *}<\omega<T_{K}$ that the leading irrelevant operator determines the dominant scaling behavior of the local fermion's susceptibility, i.e., we observe the true two-channel Kondo physics. The expected properties of $\hat{\rho}_{f}$ and the real part of its dimensionless Green's function $\hat{g}_{f}$ in the presence of channel asymmetry are summarized in Fig. 3. These analytic expectations are indeed met by our NRG results.
Fig. 4. (a) depicts the spectral function of $f_{0,1, \sigma}^{\dagger}$ for several values of $K_{R}$ as a function of $\omega / T_{K}$ on a logarithmic scale. The overall scaling is very similar to the one sketched in Fig. 3 except that the high temperature plateau is missing; this is due to the relatively large value of $T_{K}$, which is only one decade smaller than the bandwidth cut-off. Figures $4 .(b-e)$ are the numerical confirmations of the asymptotics stated. In all these figures dashed straight lines are to demonstrate deviations from the expected behavior. In Fig. 4.(b) we show the square root-like asymptotics in the $2 \mathrm{CK}$ scaling regime for the channel symmetric case. This behavior is a consequence of the dimension of the leading irrelevant operator as it has just been discussed. In Fig. 4. $(c)$ the same asymptotics is shown in the same region in case of a finite channel anisotropy, whereas below them Fig. 4. $(d)$ demonstrates $(1 / \omega)^{1 / 2}$-like behavior resulting from the relevant perturbation of the 2CK fixed point Hamiltonian with channel anisotropy. In Fig. 目. $(e)$ the FL-like $\omega^{2}$-behavior is recovered below $T^{*}$, which is typical of fermionic operators in the $1 \mathrm{CK}$ scaling regimes.

In Figs. 5 . $(a-b)$ we show the universal scaling curves, $\mathcal{K}^{ \pm}$that connect the two-channel and single channel fixed points at low-frequencies as a function of $\omega / T^{*}$. They were computed from runs with negative and positive values of $K_{R}$. This universal behavior is violated for values

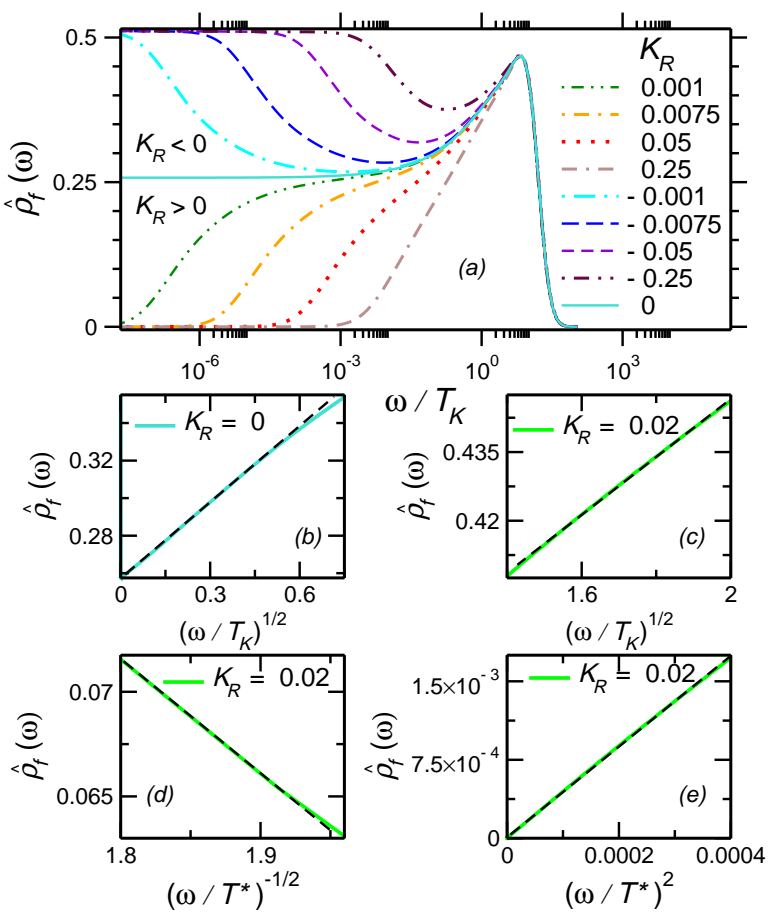

FIG. 4: (color online) (a) Dimensionless spectral function of $f_{0,1, \sigma}: \hat{\varrho}_{f}(\omega)=D_{F} \varrho_{f}(\omega)$ as a function of $\omega / T_{K}$ for different values of $K_{R}$. $(b-e)$ Numerical confirmations of the lowfrequency asymptotics derived through scaling arguments in Sec. V] Dashed straight lines are to demonstrate deviations from the expected $\sqrt{\omega}$-like $(b-c), 1 / \sqrt{\omega}$-like $(d)$ and $\omega^{2}$-like (e) behavior. In plots $(c-e) T^{*} / T_{K}=2.4 \times 10^{-4}$. 


\begin{tabular}{|c|c|c|c|}
\hline \multirow{2}{*}{ Scaling Function } & Asymptotic Form & \multirow{2}{*}{$\begin{array}{c}\text { Scaling Variable } \\
x \\
\end{array}$} & \multirow{2}{*}{$\begin{array}{c}2 \mathrm{CK} \\
\text { Scaling Regime }\end{array}$} \\
\hline & $x \ll 1$, & & \\
\hline $\begin{array}{l}\Theta_{f}(x) \\
\tilde{\Theta}_{f}(r)\end{array}$ & 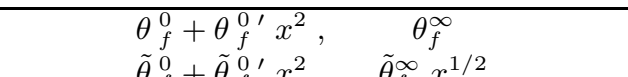 & $\omega / T$ & $T \lesssim \omega$ \\
\hline$\tilde{\Theta}_{f}(x)$ & $\begin{aligned} \tilde{\theta}_{f}^{0}+\tilde{\theta}_{f}^{0 \prime} x^{2}, & \tilde{\theta}_{f}^{\infty} x^{1 / 2} \\
\kappa^{ \pm} & \kappa^{ \pm} \quad+\left.\kappa^{ \pm \prime}\right|^{1 / 2}\end{aligned}$ & & \\
\hline $\begin{array}{l}\mathcal{K}_{f}^{ \pm}(x) \\
\tilde{\mathcal{K}}_{f}^{ \pm}(x)\end{array}$ & $\begin{array}{cc}\kappa_{f, 0}^{ \pm}+\kappa_{f, 0}^{ \pm \prime} x^{2}, & \kappa_{f, \infty}^{ \pm}+\kappa_{f, \infty}^{ \pm \prime}\left|\frac{1}{x}\right|^{1 / 2} \\
\tilde{\kappa}_{f, 0}^{ \pm}|x|^{3 / 2}, & \tilde{\kappa}_{f, \infty}^{ \pm}\end{array}$ & $\omega / T^{*}$ & $T_{f}^{* *} \lesssim \omega, \quad T_{f}^{* *} \propto \sqrt{T^{*} T_{K}}$ \\
\hline $\begin{array}{l}\mathcal{B}_{f, \sigma}(x) \\
\tilde{\mathcal{B}}_{f, \sigma}(x)\end{array}$ & 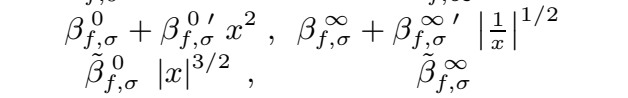 & $\omega / T_{h}$ & $T_{h}^{* *} \lesssim \omega, \quad T_{h}^{* *} \propto \sqrt{T_{h} T_{K}}$ \\
\hline $\begin{array}{l}\Theta_{S}(x) \\
\tilde{\Theta}_{S}(x)\end{array}$ & $\begin{array}{lc}\theta_{S}^{0} x, & \theta_{S}^{\infty} \operatorname{sgn}(x) \\
\tilde{\theta}_{S}^{0} x, & \tilde{\theta}_{S}^{\infty} \operatorname{sgn}(x)|x|^{1 / 2}\end{array}$ & $\omega / T$ & $T \lesssim \omega$ \\
\hline $\begin{array}{l}\mathcal{K}_{S}(x) \\
\tilde{\mathcal{K}}_{S}(x)\end{array}$ & $\begin{array}{cc}\kappa_{S}^{0} x, & \kappa_{S}^{\infty} \operatorname{sgn}(x)+\kappa_{S}^{\infty} " \frac{1}{x} \\
\tilde{\kappa}_{S}^{0} \operatorname{sgn}(x)|x|^{1 / 2}, & \tilde{\kappa}_{S}^{\infty} \operatorname{sgn}(x)\end{array}$ & $\omega / T^{*}$ & $T_{s}^{* *} \lesssim \omega, \quad T_{s}^{* *} \propto\left(T^{* 2} T_{K}\right)^{1 /}$ \\
\hline $\begin{array}{l}\mathcal{B}_{S, z}(x) \\
\tilde{\mathcal{B}}_{S, z}(x)\end{array}$ & $\begin{array}{c}\beta_{S, z}^{\infty}+\beta_{S, z}^{\infty}\left|\frac{1}{x}\right|^{1 / 2} \\
\tilde{\beta}_{S, z}^{\infty} \\
\end{array}$ & $\omega / T_{h}$ & $T_{h}^{* *} \lesssim \omega, \quad T_{h}^{* *} \propto \sqrt{T_{h} T_{K}}$ \\
\hline
\end{tabular}

TABLE IV: Asymptotic behavior of the universal cross-over functions. At finite temperature, the boundary of the two-channel Kondo scaling regime is set by the temperature. At zero temperature, the various boundaries of the $2 \mathrm{CK}$ scaling regime derive from the competition between the leading irrelevant operator and the relevant perturbation.

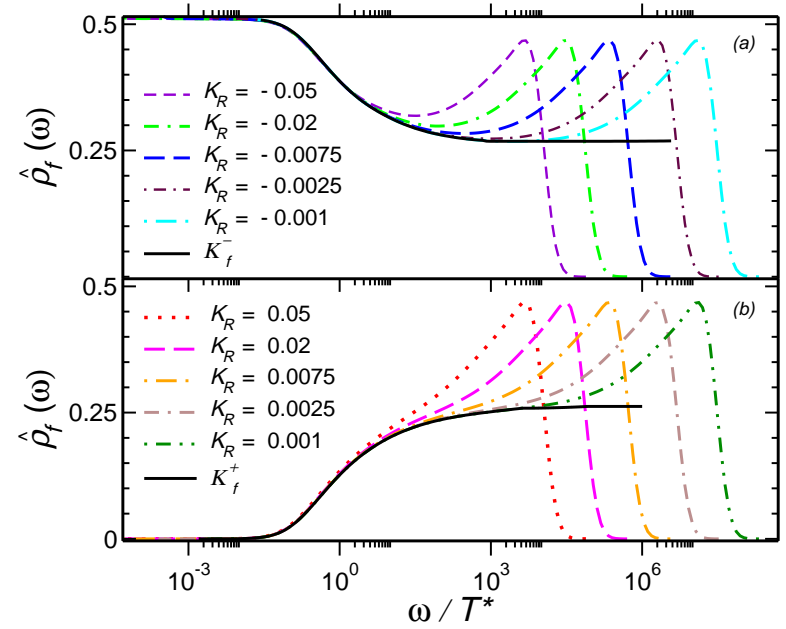

FIG. 5: (color online) Universal collapse of the dimensionless spectral functions, $\hat{\varrho}_{f}=D_{F} \varrho_{f}$ (with $f$ in channel 1) to two scaling curves, $\mathcal{K}_{f}^{ \pm}$as a function of $\omega / T^{*}$ for positive $(b)$ and negative $(a)$ values of $K_{R}$.

of $K_{R}$ higher than the highest ones shown in Fig. 5 , where $T^{*}$ becomes comparable to $T_{K}$.

The real parts of the local fermion susceptibilities are plotted in Fig. 6 for several values of $K_{R}$. They were obtained by performing the Hilbert transformations numerically. They should show a three-peak structure based on the analytic considerations (see Fig. 3). There are two low-frequency peaks clearly visible, associated with the cross-overs at $T^{*}$ and $T_{K}$. Furthermore there should be a non-universal peak at the cut-off. For relatively large channel anisotropies, where $T^{*} \sim T_{K}$, the former two peaks cannot be clearly separated in Fig. 6. Also, due to the large value of $T_{K} \sim$ the band cut-off, $D_{F}$, the peak at $\omega \sim T_{K}$ and the smeared singularity at $\omega=D_{F}$ merge to a single non-universal feature in our NRG curves.

Let us now turn to the effect of a finite magnetic field, $B \neq 0$ for the case $T=0, K_{R}=0$. As $h$ and $\kappa$ scale the same way in the $2 \mathrm{CK}$ scaling regime, the argument concerning the $\kappa \neq 0$ case can be repeated with minor modifications. Now, however, the spin $\mathrm{SU}_{S}(2)$ symmetry is violated, and therefore the spectral functions of $f_{0, \alpha, \uparrow}^{\dagger}$ and $f_{0, \alpha, \downarrow}^{\dagger}$ become different, and they are no longer even either. Nevertheless, due to particle-hole symmetry, they are still related through the relations

$$
\begin{aligned}
& \hat{\rho}_{f, \uparrow}(\omega, T, \kappa, h, \ldots)=\hat{\rho}_{f, \downarrow}(-\omega, T, \kappa, h, \ldots), \\
& \hat{\rho}_{f, \uparrow}(\omega, T, \kappa, h, \ldots)=\hat{\rho}_{f, \downarrow}(\omega, T, \kappa,-h, \ldots) .
\end{aligned}
$$

We are thus free to choose the orientation of the magnetic

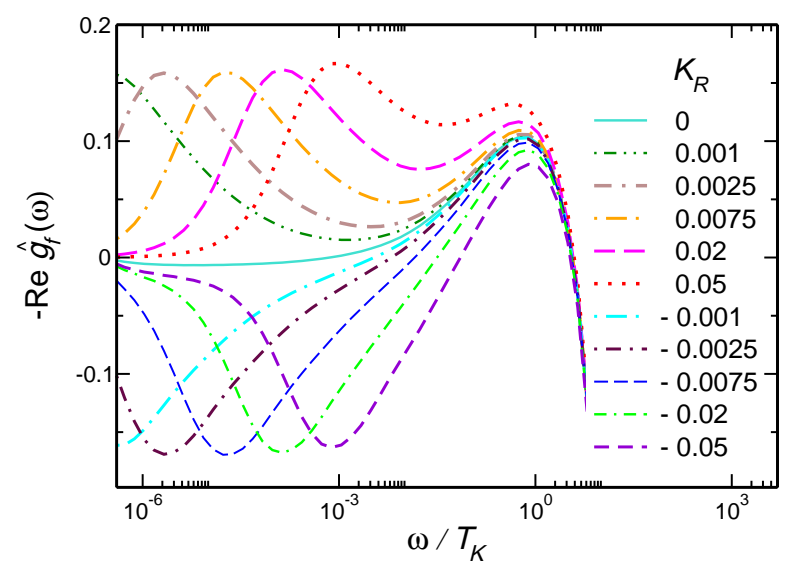

FIG. 6: (color online) Real part of the dimensionless Green's function: $\operatorname{Re} \hat{g}_{f}=D_{F} \operatorname{Re} \mathcal{G}_{f}$ (with $f^{\dagger}$ in channel 1) as a function of $\omega / T_{K}$ for different values of $K_{R}$. From among the three peaks sketched in Fig. 3 only the two peaks around $T^{*}$ and $T_{K}$ are shown. 

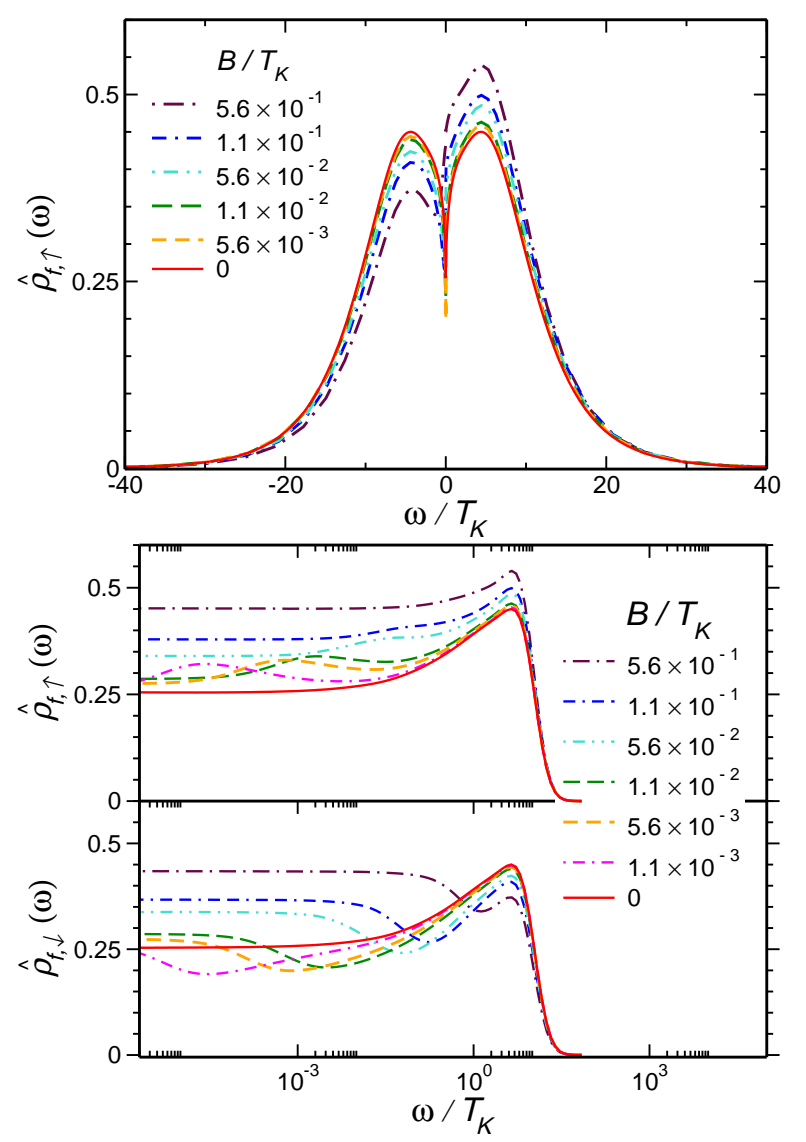

FIG. 7: (color online) Top: Dimensionless spectral function of $f_{0,1, \uparrow}: \hat{\varrho}_{f, \uparrow}=D_{F} \varrho_{f, \uparrow}$ for different values of $B$ as a function of $\omega / T_{K}$ on linear scale. Bottom: Dimensionless spectral function of $f_{0,1, \uparrow}(a)$ and of $f_{0,1, \downarrow}(b)$ for different values of $B$ as a function of $\omega / T_{K}$ on logarithmic scale.

field downwards. Then, after rescaling $D \rightarrow|\omega|$ we get

$$
\hat{\varrho}_{f, \sigma}^{\kappa, T=0}(\omega)=\mathcal{B}_{f, \sigma}\left(\frac{\omega}{T_{h}}\right)+\sqrt{\frac{|\omega|}{T_{K}}} \tilde{\mathcal{B}}_{f, \sigma}\left(\frac{\omega}{T_{h}}\right)+\ldots
$$

where the label $\sigma$ refers to the different spin components and $\mathcal{B}_{f, \sigma}$ and $\tilde{\mathcal{B}}_{f, \sigma}$ are yet another pair of universal crossover functions. The asymptotic properties of the functions $\mathcal{B}_{f, \sigma}$ and $\tilde{\mathcal{B}}_{f, \sigma}$ are summarized in Table $\mathbf{V}$

Fig. 7 shows the spectral functions $\hat{\varrho}_{f, \sigma}$ as a function of $\omega / T_{K}$ on linear and logarithmic scales for different magnetic field values. The same curves are depicted as a function of $\omega / T_{h}$ in Fig. 8, which demonstrates the existence of the universal scaling curves, $\mathcal{B}_{f, \sigma}$, i.e. that by using the scale, $T_{h}$ the local fermion's spectral functions can be scaled on top of each other for small enough magnetic fields. In this magnetic field region, we find a peak at $T_{h}$ for the spin- $\uparrow$ component of $f^{\dagger}$, while at the same place there is a dip for the spin- $\downarrow$ component. This is a remarkable feature that is associated with inelastic scattering off the slightly polarized impurity spin. In fact,

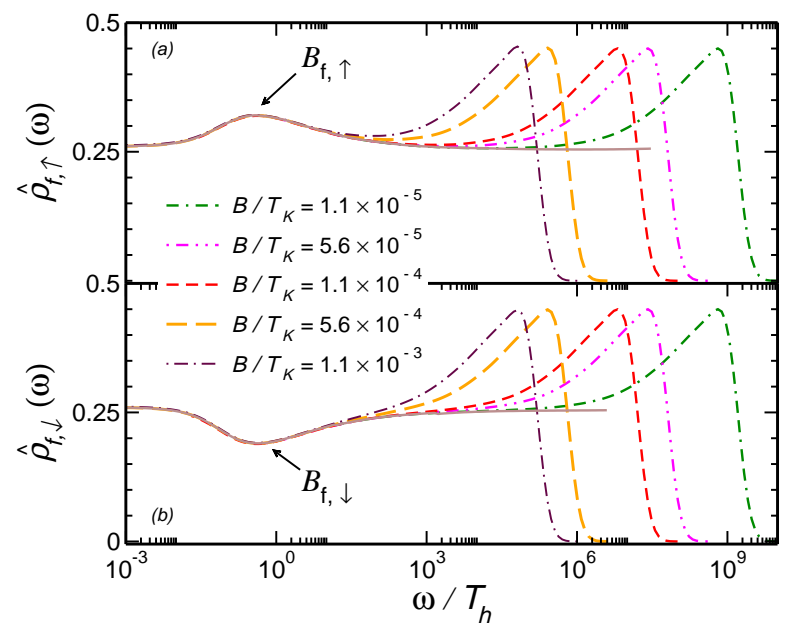

FIG. 8: (color online) Universal collapse of the dimensionless spectral functions: $\varrho_{f, \uparrow}=D_{F} \varrho_{f, \uparrow}$ and $\hat{\varrho}_{f, \downarrow}=D_{F} \varrho_{f, \downarrow}$ to two scaling curves: $\mathcal{B}_{f, \uparrow}$ and $\mathcal{B}_{f, \downarrow}$ for sufficiently small, non-zero values of $B$ as a function of $\omega / T_{h}$.

the same uinversal features also appear in the spectral functions of the composite fermions, which we compute independently and which are directly related to those of the conduction electrons by Eqn. (30) $\stackrel{46}{\text { The rescaled }}$ spectral functions $\hat{\varrho}_{F, \sigma}(\omega)$ are shown in Fig 9.

Although this numerical evidence can be obtained by conventional NRG methods not using the density matrix, this is no longer true for the sum of the local fermions spectral function over the different spin components. In fact, for this quantity universal scaling curves in the presence of magnetic field cannot be obtained using NRG because of the increase in the size of the numerical errors at

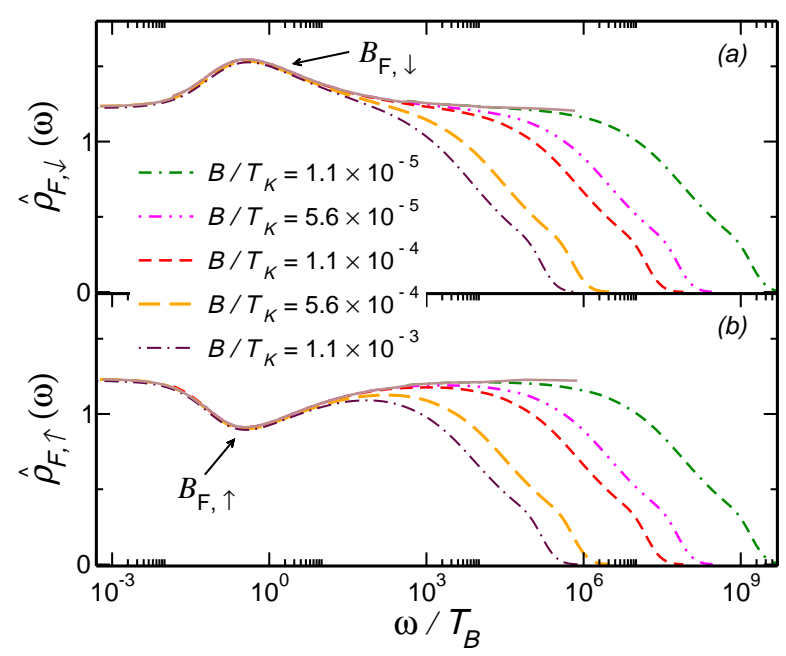

FIG. 9: (color online) Universal collapse of the dimensionless spectral functions of the composite fermion operator: $\hat{\varrho}_{F, \uparrow}=$ $D_{F} \varrho_{f, \uparrow}$ and $\hat{\varrho}_{F, \downarrow}=D_{F} \varrho_{F, \downarrow}$ to two scaling curves: $\mathcal{B}_{F, \uparrow}$ and $\mathcal{B}_{F, \downarrow}$ for sufficiently small, non-zero values of $B$ as a function of $\omega / T_{h}$. 


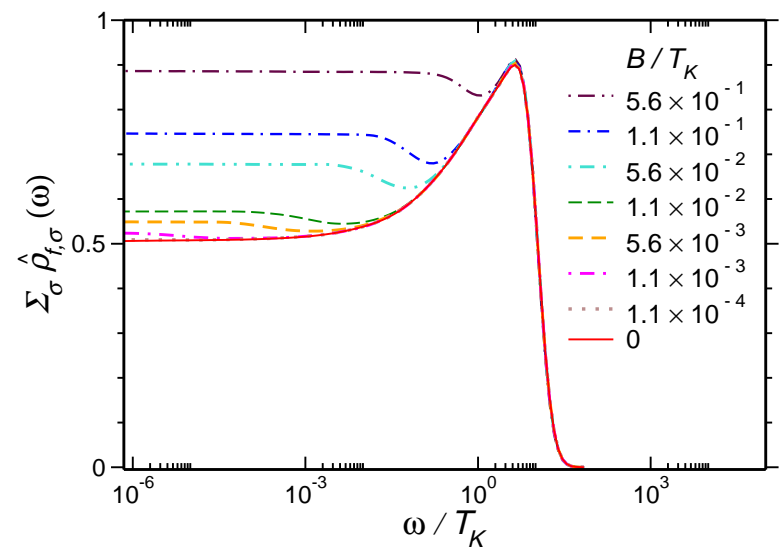

FIG. 10: (color online) Sum of the dimensionless spectral functions: $\hat{\varrho}_{f, \uparrow}=D_{F} \varrho_{f, \uparrow}$ and $\hat{\varrho}_{f, \downarrow}=D_{F} \varrho_{f, \uparrow}$ for different values of $B$ as a function of $\omega / T_{K}$.

low-frequencies and the mismatch between the positive and negative frequency parts of the spectral functions. The sum of the local fermion's spectral function over the two spin components is depicted in Fig. [10 as a function of $\omega / T_{K}$. Here the splitting of the Kondo resonance in the energy-dependent scattering cross section appears as a minimum at $\omega \sim T_{h}$. Unfortunately, for even smaller magnetic fields the accuracy of our numerical data is insufficient to tell if the splitting of the Kondo resonance survives in the limit $B \rightarrow 0$, as conjectured in Ref. 24 . In the data with $B / T_{K}>1.1 \times 10^{-4}$, there seems to be always a shallow minimum in the spectral function, and we see no indication for crossing of the curves as the magnitude of the field is reduced. If there is indeed no crossing of the spectral functions and if the deviation from the $\sqrt{|\omega|}$-behavior indeed starts at $\omega \approx T_{h} \sim B^{2} / T_{K}$, which is the only natural assumption, then, from exact Bethe Ansatz results it would immediately follow that there must always be a splitting of the Kondo resonance, since $\sum_{\sigma}\left[\varrho_{f \sigma}(\omega=0, B)-\varrho_{f \sigma}(\omega=0,0)\right] \sim B \ln \left(T_{K} / B\right) \stackrel{22}{,}$ while $\sum_{\sigma}\left[\varrho_{f \sigma}\left(\omega=T_{h}, B\right)-\varrho_{f \sigma}(\omega=0,0)\right] \sim|B|$ would follow from the pure $\sqrt{|\omega|}$-dependence of the spectral function at $B=0$. However, these analytical arguments do not constitute a real proof.

With small modifications, the analysis presented in this subsection carries over to essentially any fermionic operator that has quantum numbers $c_{1}=j=1 / 2$ or $c_{2}=j=1 / 2$ and has a finite overlap with the primary fields $\phi_{\psi 1}$ and $\phi_{\psi 2}$, only the high-frequency behavior $\left(\omega>T_{K}\right)$ and the normalization factors become different. Typically, a local operator having the same charge and spin quantum numbers as $\phi_{\psi \alpha}$ will have a finite overlap with them. However, in some cases the internal Ising quantum number of an operator may prevent an overlap and, of course, one can also construct operators by, say, differentiating with respect to the time, that would correspond to descendant fields.

\section{SPIN SPECTRAL FUNCTIONS AND SUSCEPTIBILITIES}

In this section, we shall discuss the properties of the spin operator, $\vec{S}$, which is the most obvious example of a bosonic operator of spin $j=1$ and charge quantum numbers $c_{1}=c_{2}=0$ that overlaps with the scaling operator $\phi_{s}$. There are, however, many operators that have the same quantum numbers: two examples are the so-called channel spin operator,

$$
\vec{S}_{C} \equiv f_{0,1}^{\dagger} \vec{\sigma} f_{0,1}-f_{0,2}^{\dagger} \vec{\sigma} f_{0,2},
$$

or a composite channel spin operator

$$
\vec{S}_{C C} \equiv F_{0,1}^{\dagger} \vec{\sigma} f_{0,1}-F_{0,2}^{\dagger} \vec{\sigma} f_{0,2} .
$$

Our discussion can be easily generalized to these operators with minor modifications.

The analysis of the spin spectral function goes along the lines of the previous subsection. First we recall that the field $\vec{\phi}_{s}$ appears in the expansion of the spin operator,

$$
\vec{S}=A_{s} \vec{\phi}_{s}+\ldots,
$$

with $A_{s} \sim 1 / \sqrt{T_{K}} \sim 1 / \sqrt{D_{0}}$. Therefore, the appropriate dimensionless scale invariant Green's function (usually referred to as the dynamical spin susceptibility) is defined as

$$
\hat{g}_{S}\left(\frac{\omega}{D}, \frac{T}{D}, \kappa_{0}, h_{0}, \ldots\right) \equiv T_{K} G_{S}\left(\omega, T, \kappa_{0}, \ldots, D_{0}\right) .
$$

We shall not repeat here all the steps of the derivation, only summarize the main results. In the absence of a magnetic field, $h=0$ the spectral function of the spin operator is odd. Furthermore, at $T=0$ and for no anisotropy, $\kappa=0$, the spectral function has a jump at $\omega=0, \frac{5}{5}$

$$
\hat{\varrho}_{S}^{T, h, \kappa=0}(\omega) \approx \operatorname{sgn}(\omega)\left[r_{S}+r_{S}^{\prime} \sqrt{\frac{|\omega|}{T_{K}}}+\ldots\right] .
$$

This jump corresponds to a logarithmically divergent dynamical susceptibility, $\operatorname{Re} \chi_{S}(\omega)=-\operatorname{Re} \mathcal{G}_{S}(\omega) \propto$ $\ln \left(T_{K} / \omega\right) / T_{K}$.

For $\omega \gg T_{K}$ the impurity spin becomes asymptotically free, decoupled from the conduction electrons, therefore its $\omega$-dependence is set by its scaling dimension at the free fermion fixed point where $x_{S}^{f r e e}=0$. It has the implication that its correlation fuction decays as $\omega^{-1}$ corresponding to the Curie-Weiss susceptibility with logarithmic corrections present, known from Bethe Ansatz results and from perturbation theory.

At finite temperatures $T \neq 0$, but for $\kappa=h=0$, we obtain the following scaling form for $T, \omega \ll T_{K}$ :

$$
\hat{\varrho}_{S}^{h, \kappa=0}(\omega) \equiv \Theta_{S}\left(\frac{\omega}{T}\right)+\sqrt{\frac{T}{T_{K}}} \tilde{\Theta}_{S}\left(\frac{\omega}{T}\right)+\ldots
$$




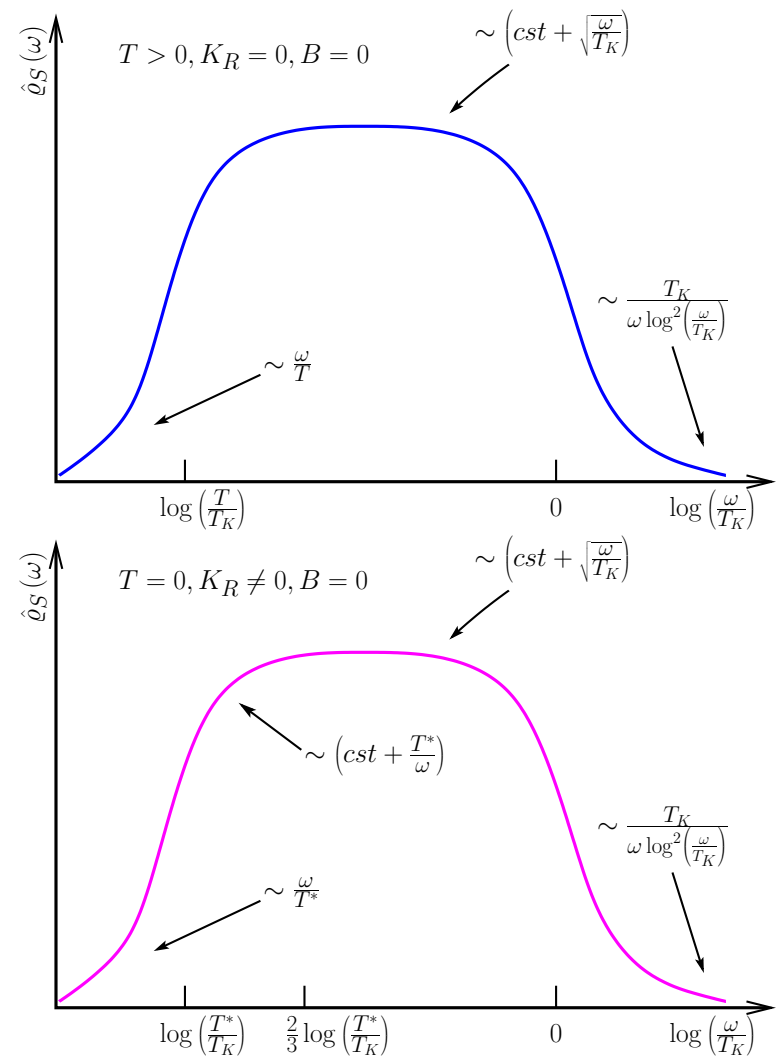

FIG. 11: (color online) Top: Sketch of the dimensionless spectral function of $\vec{S}: \hat{\varrho}_{S}=T_{K} \varrho_{S}=-T_{K} \operatorname{Im} \chi_{S}(\omega) / \pi$ for $T>0$ and $K_{R}=0, B=0$ as a function of $\log \left(\omega / T_{K}\right)$. Bottom: Sketch of $\hat{\varrho}_{S}=T_{K} \varrho_{S}=-T_{K} \operatorname{Im} \chi_{S}(\omega) / \pi$ for $T=0$ and $K_{R} \neq 0, B=0$ as a function of $\log \left(\omega / T_{K}\right)$. Asymptotics indicated for $\omega<T_{K}$ were derived through scaling arguments. The large $\omega$-behavior is a result of perturbation theory: ${ }^{48}$

The asymptotic properties of the scaling functions $\Theta_{S}$ and $\tilde{\Theta}_{S}$ are listed in Table $\mathrm{V}$

In case of finite channel anisotropy but zero temperature we obtain for $\omega \ll T_{K}$ the scaling form

$$
\hat{\varrho}_{S}^{T, h=0}(\omega) \approx \mathcal{K}_{S}\left(\frac{\omega}{T^{*}}\right)+\sqrt{\frac{|\omega|}{T_{K}}} \tilde{\mathcal{K}}_{S}\left(\frac{\omega}{T^{*}}\right)+\ldots .
$$

The asymptotic properties of $\mathcal{K}_{S}, \tilde{\mathcal{K}}_{S}$ are only slightly different from those of $\Theta_{S}, \tilde{\Theta}_{S}$ (see Table $\nabla$ ): below $T^{*}$ the spectral function displays analytic behavior, while the regime $\omega>T^{*}$ is governed by non-analytical corrections associated with the $2 \mathrm{CK}$ fixed point. In this regime a feature worth mentioning is the appearance of a correction, $\sim T^{*} / \omega$ to $\mathcal{K}_{S}$, more precisely, the lack of a $\sqrt{\left|T^{*} / \omega\right|}$ correction. This is due to the fact that the anisotropy operator is odd, while the spin operator is even with respect to swapping the channel labels. Therefore there is no first order correction to the spin-spin correlation function in $\kappa$, and the leading corrections are only of second order, i.e., of the form $\kappa^{2} / \omega$. From the comparison of the terms in $\mathcal{K}_{S}$ and $\tilde{\mathcal{K}}_{S}$ it also follows the existence of

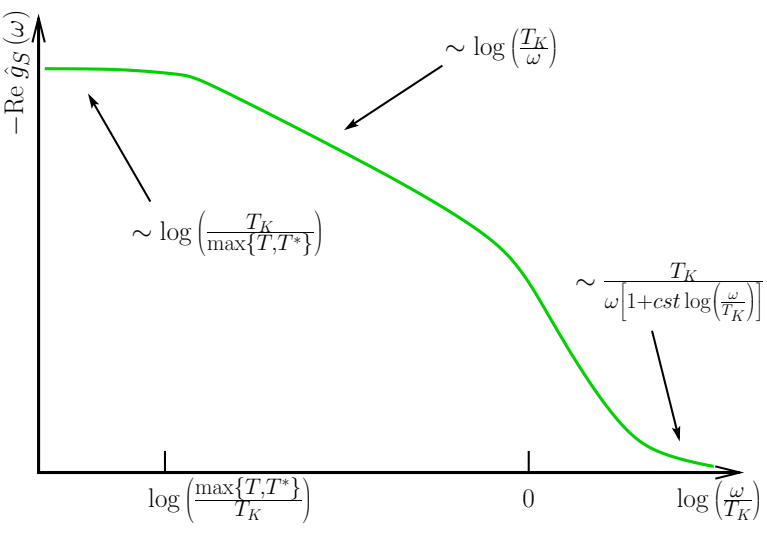

FIG. 12: (color online) Sketch of the real part of the dimensionless Green's function of $\vec{S}$, Re $\hat{g}_{S}=T_{K} \operatorname{Re} \chi_{S}(\omega) \equiv$ $T_{K} \operatorname{Re} \mathcal{G}_{S}(\omega)$ for $T, T^{*}>0$ as a function of $\log \left(\omega / T_{K}\right)$.

another cross-over scale,

$$
T_{s}^{* *} \sim\left(T^{* 2} T_{K}\right)^{1 / 3}
$$

that separates the regimes governed by the leading relevant and leading irrelevant operators. Here we used the subscript $s$ to indicate that this scale $T_{s}^{* *}$ is different from the scale $T_{f}^{* *}$ introduced in relation to the local fermion's spectral function. The asymptotic properties of $\hat{\varrho}_{S} \propto \chi_{S}(\omega)$ for $T>0, K_{R}=0$ and $T=0, K_{R} \neq 0$ are sketched in the upper and lower parts of Fig. 11, while the behavior of the real part is presented in Fig. 12

The expectations above are indeed nicely born out by the NRG calculations: Fig. 13 shows the impurity spin spectral functions as a function of $\omega / T_{K}$ for various $K_{R^{-}}$ $\mathrm{s}$ and their asymptotic properties. First, in Fig. 13. $(b)$ we show a very small logarithmic $\omega$-dependence that we observed below $T_{K}$ at the $2 \mathrm{CK}$ fixed point. The amplitude of this $\log (\omega)$-dependence was reduced as we increased the number of multiplets. It appears that this behavior is not derived from the lognormal smoothing of the NRG data, and it may be due to some approximations used in the spectral sum-conserving DM-NRG procedure. In Fig. 13 $(c)$ we show the square root-like behavior around the 2CK Kondo fixed point which is attributed to the leading irrelevant operator, while Fig. 13 ( $(d)$ shows that first order corrections coming from the scaling of the channel anisotropy are indeed absent just as we stated above, and only second order terms appear, resulting in an $1 / \omega$-like behavior. Finally, Fig. [13. $(e)$ demonstrates the linear $\omega$-dependence, which is characteristic of most bosonic operators in the proximity of an FL fixed point. All these findings support very nicely the analytical properties summarized in Table $\mathrm{V}$.

The spin spectral functions also collapse to a universal scaling curve describing the cross-over from the twochannel Kondo to the single channel Kondo fixed points, when they are plotted against $\omega / T^{* *}$. This universal data collapse is demonstrated in Fig. 14 where the impurity 

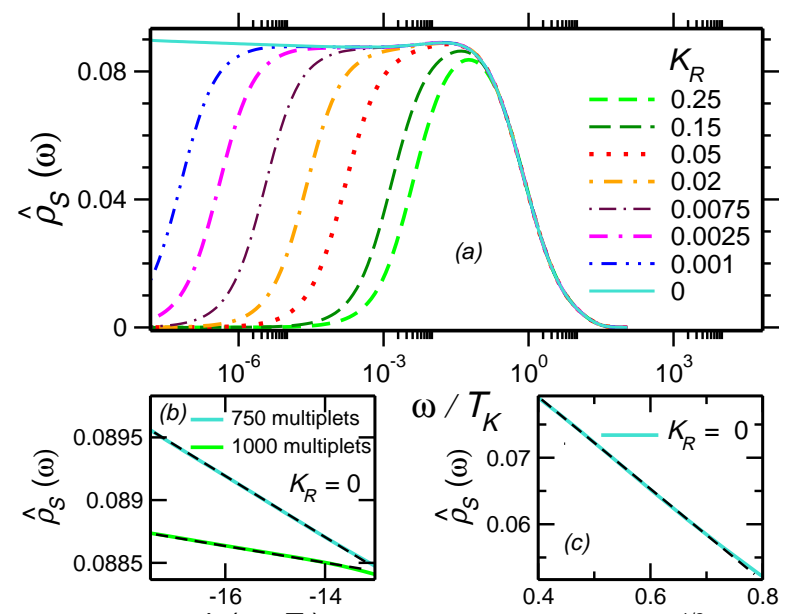

$\ln \left(\omega / T_{K}\right)$
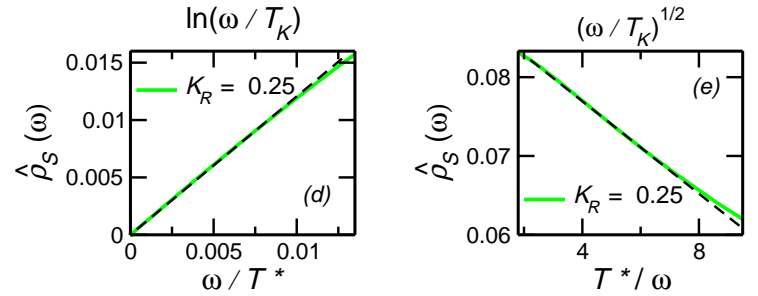

FIG. 13: (color online) (a) Dimensionless spectral function of $\vec{S}: \hat{\varrho}_{S}=T_{K} \varrho_{S}=-T_{K} \operatorname{Im} \chi_{S}(\omega) / \pi$ as a function of $\omega / T_{K}$ for different values of $K_{R}$. (b) Minute $\log (\omega)$-dependence at the lowest frequencies diminishing as a function of the number of kept multiplets. $(c-e)$ Numerical confirmations of the low-frequency asymptotics derived from scaling arguments in Section VI Straight dashed lines are to demonstrate deviations from the expected $\sqrt{\omega}$-like $(c), \omega$-like $(d)$, and $1 / \omega$-like behavior $(e)$. In plots $(d-e) T^{*} / T_{K}=7 \times 10^{-2}$.

spin spectral functions are plotted for various $K_{R}$ values. The data collapse works up to somewhat higher anisotropy values than for the local fermions' spectral functions as it is indicated by the $K_{R^{-}}$-dependence of the scales $T_{s}^{* *}$ and $T_{f}^{* *}$.

The real part of the spin susceptibility was obtained through numerical Hilbert transformation, and is shown in Fig. 15] as a function of $\omega / T_{K}$ for various values of $K_{R}$. These curves meet the expected behavior sketched in Fig. 12, they display a logarithmic increase at highfrequencies and saturate at values that correspond to $\operatorname{Re} \chi_{S} \sim \ln \left(T_{K} / T^{*}\right) / T_{K}$.

Let us finally discuss the case, $T=\kappa=0$ but $h \neq$ 0 . Then the components of $\vec{S}$ are distinguished by the magnetic field: The spectral function of $S^{z}$ has almost the same features as for finite channel anisotropies. Since $S^{z}$ is a hermitian operator, its spectral function remains odd and acquires the following corrections in the different scaling regimes

$$
\hat{\varrho}_{S, z} \equiv \mathcal{B}_{S, z}\left(\frac{\omega}{T_{h}}\right)+\sqrt{\frac{|\omega|}{T_{K}}} \quad \tilde{\mathcal{B}}_{S, z}\left(\frac{\omega}{T_{h}}\right)+\ldots
$$

with the scaling functions $\mathcal{B}_{S, z}, \tilde{\mathcal{B}}_{S, z}$ having the asymp-

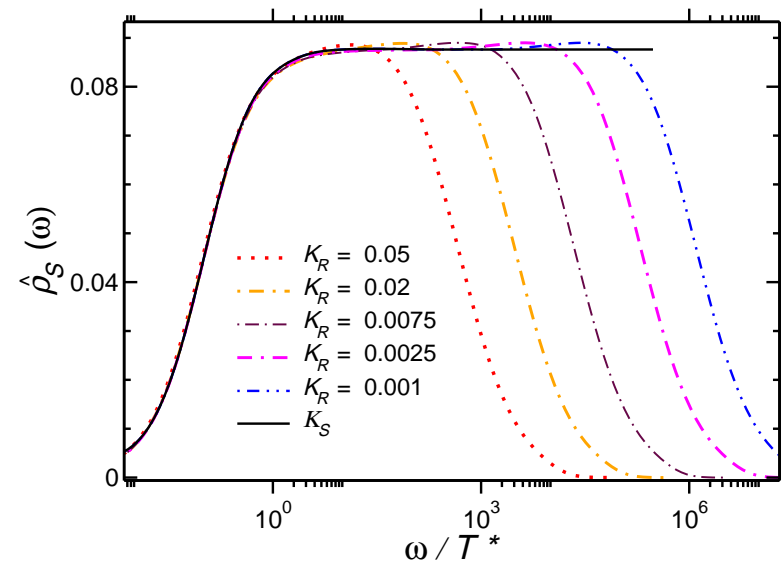

FIG. 14: (color online) Universal collapse of the dimensionless spectral function of $\vec{S}: \hat{\varrho}_{S}=T_{K} \varrho_{S}$ to the scaling curve, $\mathcal{K}_{S}$ as a function of $\omega / T^{*}$ for sufficiently small, non-zero values of $K_{R}$.

totic properties listed in Table $\mathrm{V}$.

Note that in this case the first order correction coming from the magnetic field does not vanish, and leads to the appearance of a cross-over scale $\sim \sqrt{T_{h} T_{K}}$.

The perpendicular components of the impurity spin have somewhat different properties. First of all, the operators $S^{ \pm}$are not Hermitian, and therefore their spectral functions are not symmetrical. The spectral functions of the operators $S^{x}$ and $S^{y}$ are, however, symmetrical, and their Green's functions (and susceptibilities) are related through

$$
\mathcal{G}_{S}^{x}=\mathcal{G}_{S}^{y}=\frac{1}{4}\left(\mathcal{G}_{S}^{+-}+\mathcal{G}_{S}^{-+}\right) .
$$

The corresponding dimensionless spectral functions, $\hat{\varrho}_{s}^{z}$ and $\varrho_{s}^{ \pm}$as computed by our DM-NRG calculations are shown in Fig. 16 as a function of $\omega / T_{K}$, while the universal scaling with $\omega / T_{h}$ is confirmed for low-frequencies

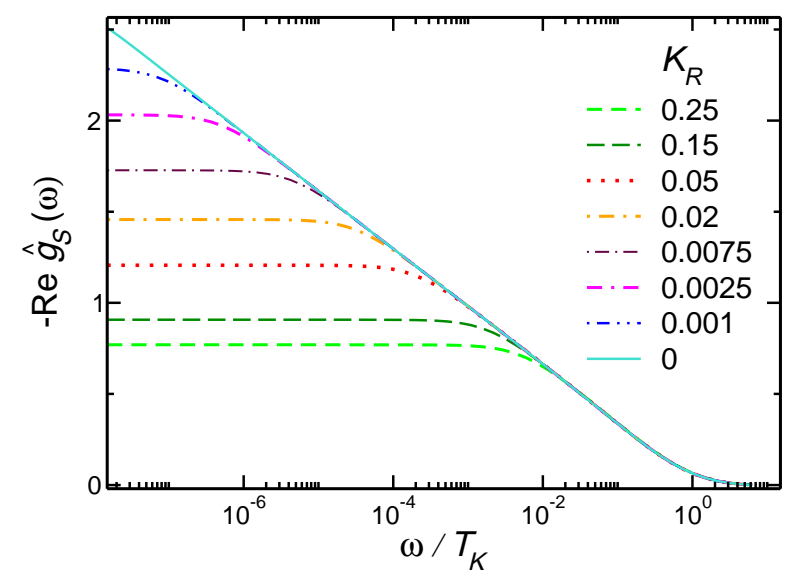

FIG. 15: (color online) Real part of the dimensionless Green's function (susceptibility) of $\vec{S}$, Re $\hat{g}_{S}=-T_{K} \operatorname{Re} \chi_{S}(\omega)$, as a function of $\omega / T_{K}$, for different values of $K_{R}$. 


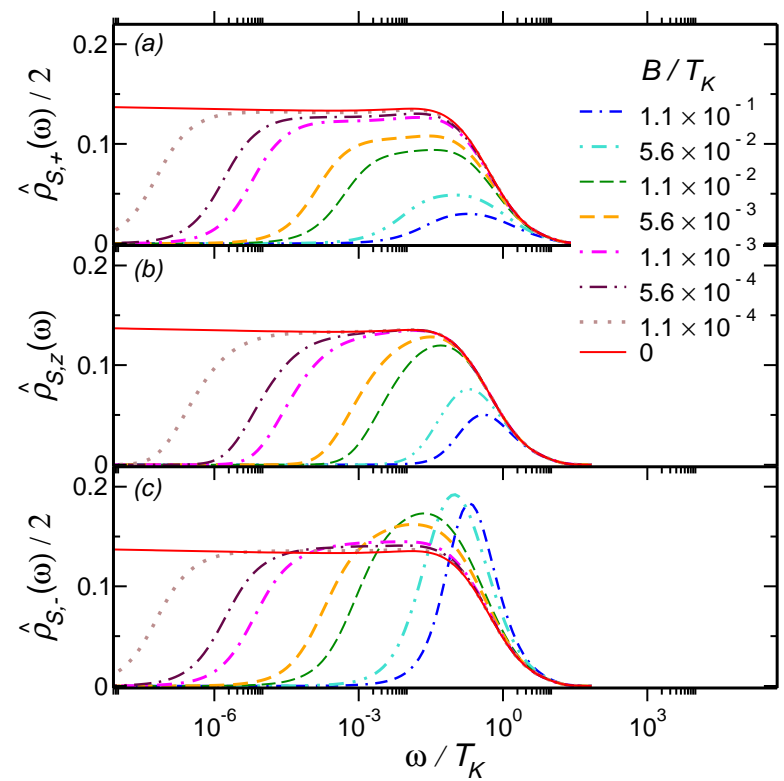

FIG. 16: (color online) (a) Dimensionless spectral function of $S^{+}: \hat{\varrho}_{S,+}=T_{K} \varrho_{S,+},(b)$ of $S^{z}: \hat{\varrho}_{S, z}=T_{K} \varrho_{S, z}$ and $(c)$ of $S^{-}: \hat{\varrho}_{S,-}=T_{K} \varrho_{S,-}$ for different values of $B$ as a function of $\omega / T_{K}$.

in Fig. 17. This scaling also turned out to be valid for values of $B$ higher than the ones for fermions (see Fig. 17). The scaling functions $\mathcal{B}_{S, z}$ and $\mathcal{B}_{S, \pm}$ behave very similarly. This is somewhat surprising, since the naive expectation would be to have a resonance in $\mathcal{B}_{S,+}$, just as in the local fermion's spectral function, that would correspond to a spin-flip excitation at the renormalized spin splitting, $T_{h}$.

excitations".

However, quite remarkably, a resonance seems to appear in $\chi_{S, z}^{\prime \prime}(\omega) / \omega$ at a frequency $\omega \sim T_{h}$, while we find no resonance in $\chi_{S, \pm}(\omega) / \omega$. This can be seen in Fig. 18, where $T_{K}^{2} \varrho(\omega) / \omega$ is plotted for the different spin components as a function of $\omega / T_{K}$ for various magnetic field values. This seems to indicate that the spin coherently oscillates between the spin up and spin down components, while its $x, y$ components simply relax to their equilibrium value.

\section{SUPERCONDUCTING CORRELATIONS}

In the last section, let us investigate the local superconducting correlation functions. These deserve special attention, since many heavy fermion compounds display exotic superconducting phases that may possibly be induced by local two-channel Kondo physics. $\stackrel{5}{5}$ The most obvious candidates for the corresponding local operators have been identified in Section III, and are the local channel-asymmetric superconducting operator, $\mathcal{O}_{S C}=$ $f_{0,1, \uparrow}^{\dagger} f_{0,2, \downarrow}^{\dagger}-f_{0,1, \downarrow}^{\dagger} f_{0,2, \uparrow}^{\dagger}$, and the composite fermion su-

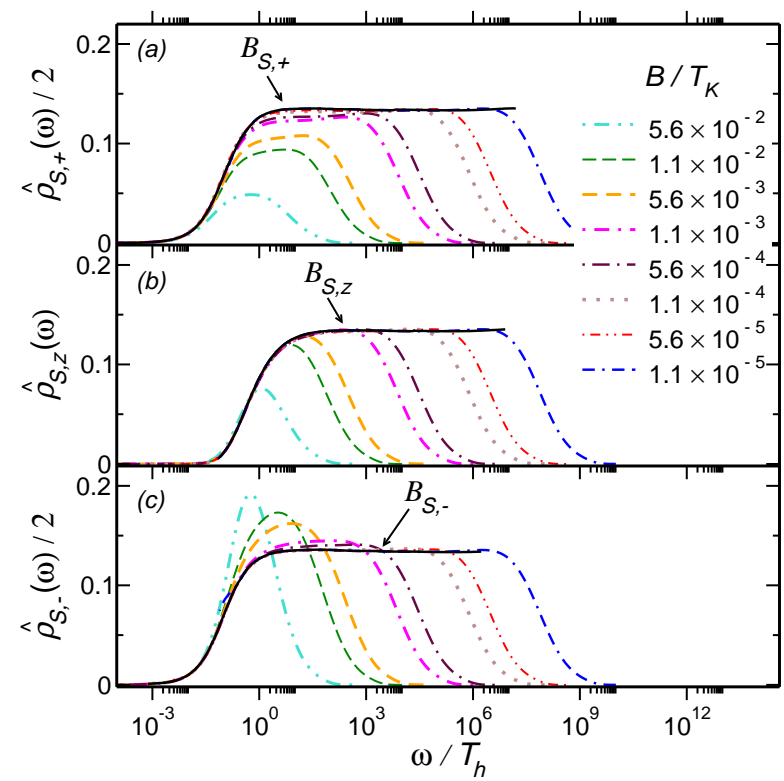

FIG. 17: (color online) Universal collapse of $\hat{\varrho}_{S,+}=T_{K} \varrho_{S,+}$, $\varrho_{S, z}=T_{K} \varrho_{S, z}$ and $\varrho_{S,-}=T_{K} \varrho_{S,-}$ to the three scaling curves: $\mathcal{B}_{S,+}, \mathcal{B}_{S, z}$ and $\mathcal{B}_{S,-}$ for sufficiently small, non-zero values of $B$ as a function of $\omega / T_{h}$.

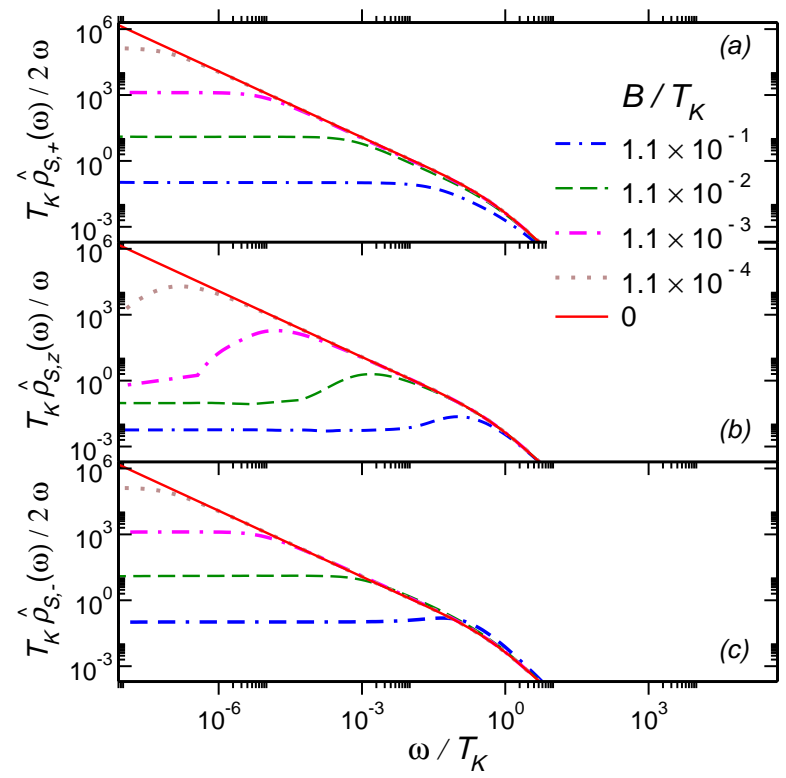

FIG. 18: (color online) (a) $\varrho(\omega) / \omega$ of $S^{+}: T_{K} \hat{\varrho}_{S,+} / 2 \omega=$ $T_{K}^{2} \varrho_{S,+} / 2 \omega,(b)$ of $S^{z}: T_{K} \hat{\varrho}_{S, z} / \omega=T_{K}^{2} \varrho_{S, z} / \omega$ and $(c)$ of $S^{-}$: $T_{K} \hat{\varrho}_{S,-} / 2 \omega=T_{K}^{2} \varrho_{S,-} / 2 \omega$ for different values of $B$ as a function of $\omega / T_{K}$.

perconductor field, $\mathcal{O}_{S C C}=f_{0,1}^{\dagger} \vec{S} \vec{\sigma} i \sigma_{y} f_{0,2}^{\dagger}$.

For the composite superconductor we find the expansion,

$$
\mathcal{O}_{S C C}=A_{S C C} \phi_{\Delta}^{++}+\ldots
$$

where the expansion coefficient $A_{S C C}$ can be estimated from the high-frequency behavior of the correlation func- 
tion up to logarithmic prefactors as $A_{S C C} \sim \sqrt{T_{K}} / D_{F}$. While for the impurity spin, one can exclude logarithmic corrections to the expansion coefficient $A_{S}$ in Eq. 46 based upon the exact Bethe Ansatz results, this is not possible for the superconducting correlation function. In fact, we know that in the expansion of the composite fermion itself the correct prefactor is $A_{F} \sim J / \sqrt{T_{K}} \sim$ $1 /\left(\sqrt{T_{K}} \ln \left(D_{F} / T_{K}\right)\right) \underline{\underline{46}}$ Therefore, similar logarithmic factors could appear in the prefactor $A_{S C C}$. Nevertheless, in the following, we shall disregard possible logarithmic corrections, and define the normalized dimensionless and scale-invariant correlation function through the relation,

$$
\frac{D_{F}^{2}}{T_{K}} \mathcal{G}_{S C C}(\omega)=\hat{g}_{S C C}(\omega)
$$

Apart from its overall amplitude and its high-frequency behavior, in the low-frequency scaling regimes the spectral function of the composite superconductor operator behaves the same way as that of $S^{z}$ (see Tab. III). Therefore we merely state its asymptotics without further explanation.

In the absence of anisotropy and magnetic field, $\kappa=$ $h=0$, for $\omega \ll T_{K}$ the spectral function becomes a universal function, $\hat{\rho}_{S C C}(\omega / T)$, whose behavior is described by the scaling form,

$$
\hat{\varrho}_{S C C}^{h, \kappa=0}(\omega) \approx \Theta_{S C C}\left(\frac{\omega}{T}\right)+\sqrt{\frac{T}{T_{K}}} \tilde{\Theta}_{S C C}\left(\frac{\omega}{T}\right)+\ldots,
$$

while in the presence of anisotropy, but at $T=0$ temperature and for $h=0$, the spectral functions behave as

$$
\hat{\varrho}_{S C C}^{T, h=0}(\omega) \approx \mathcal{K}_{S C C}\left(\frac{\omega}{T^{*}}\right)+\sqrt{\frac{|\omega|}{T_{K}}} \tilde{\mathcal{K}}_{S C C}\left(\frac{\omega}{T^{*}}\right)+\ldots
$$

Finally, in a finite magnetic field but for $\kappa=0$ anisotropy and $T=0$ temperature the spectral function assumes the following scaling form,

$$
\hat{\varrho}_{S C C}^{\kappa=T=0} \equiv \mathcal{B}_{S C C}\left(\frac{\omega}{T_{h}}\right)+\sqrt{\frac{|\omega|}{T_{K}}} \tilde{\mathcal{B}}_{S C C}\left(\frac{\omega}{T_{h}}\right)+\ldots
$$

The properties of the the various scaling functions defined above are identical to those of the corresponding spectral functions of the $S^{z}$, which were detailed in Table $\mathrm{V}$ therefore they have not been included in Table $\mathrm{V}$.

The asymptotic properties are nicely confirmed by our NRG calculations. The dependence on the anisotropy, together with the $\sim \sqrt{|\omega|}$, the $\sim 1 / \omega$ and the $\sim \omega$ scaling regimes are plotted in Fig. 19, Here the high-frequency region, $\omega>T_{K}$, is also displayed, where the spectral function is roughly linear in the frequency, as dictated by the free fermion fixed point.
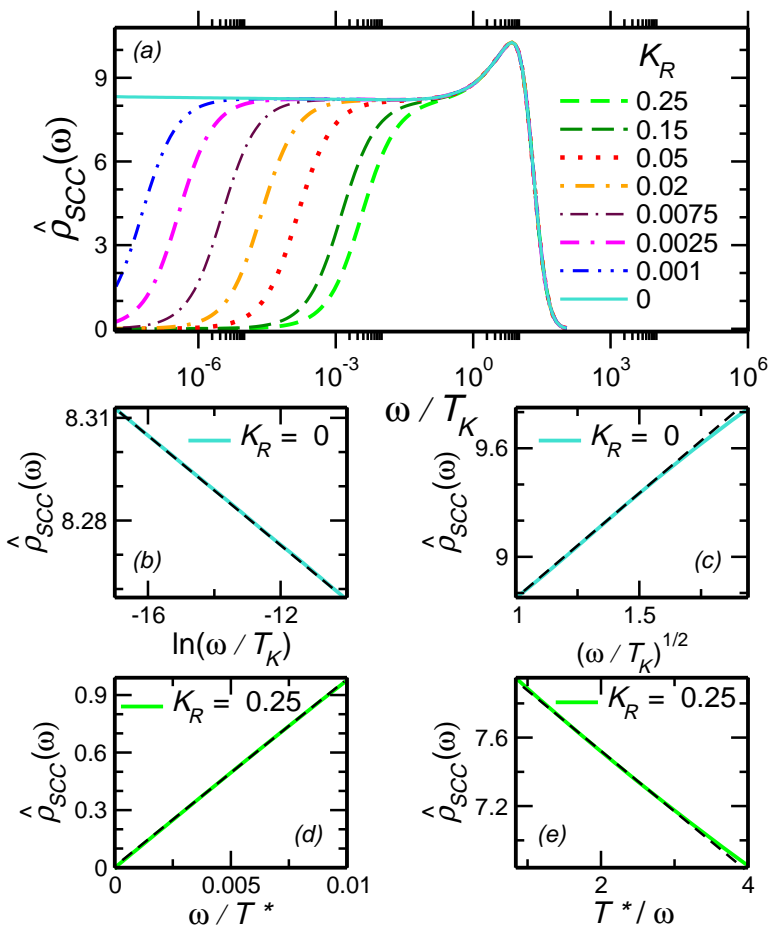

FIG. 19: (color online) (a) Dimensionless spectral function, $\varrho_{S C C}=D_{F}^{2} / T_{K} \varrho_{S C C}$ of the operator $\mathcal{O}_{S C C}$, as a function of $\omega / T_{K}$ for different values of $K_{R}$. (b). The very weak $\log (\omega)$ dependence at the lowest frequencies. This dependence is suppressed as we increased the number of kept multiplets. $(c-e)$ Numerical confirmations of the low-frequency asymptotics derived from scaling arguments in Section VII Dashed straight lines are to demonstrate deviations from the expected $\sqrt{\omega}$ like $(c), \omega$-like $(d)$ and $1 / \omega$-like $(e)$ behavior. In plots $(d-e)$ $T^{*} / T_{K}=7 \times 10^{-2}$.

Fig. 20 displays the real part of the dimensionless Green's function, that is essentially the real part of the superconducting susceptibility. This diverges logarithmically for $T^{*}=0$, but for finite $T^{*}$ 's it saturates, corresponding to a susceptibility value

$$
\chi_{S C C} \sim \frac{T_{K}}{D_{F}^{2}} \ln \left(\frac{T_{K}}{T^{*}}\right) .
$$

Notice that there is a small prefactor in front of the logarithm that arises from the asymptotically free behavior at large frequencies.

The universal collapse of the low-frequency part of the curves in terms of $\omega / T^{*}$ is shown in Fig. 21] The crossover curve, $\mathcal{K}_{S C C}\left(\frac{\omega}{T^{*}}\right)$ is very similar to the spin crossover function, $\mathcal{K}_{S}$, and displays a plateau at large frequencies from which it deviates as $1 / \omega$, until it finally reaches the linear frequency regime below $T^{*}$.

Application of a magnetic field has effects very similar to the anisotropy, as shown in the upper part of Fig. 22. In Fig. 22 the small logarithmic increase at small frequencies is more visible. As mentioned before, this increase is most likely an artifact of the spectral sum conserving approximation of Ref. 41 and it is due to the 


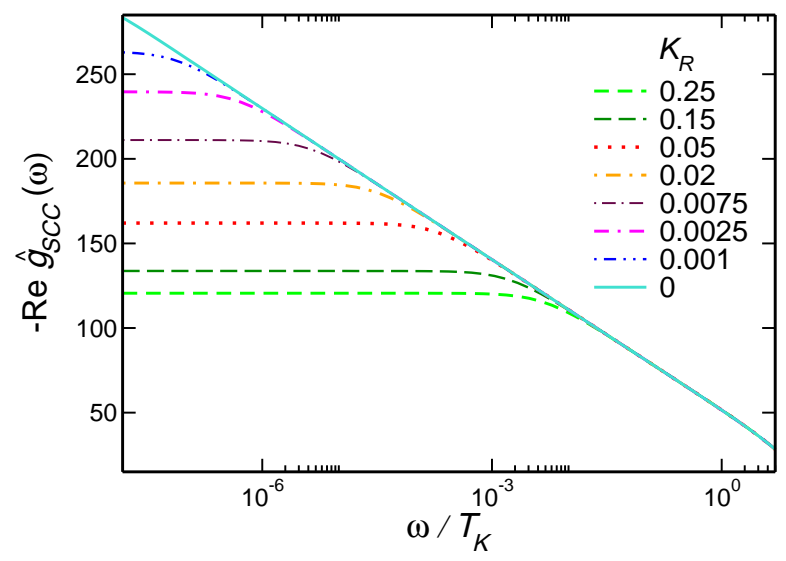

FIG. 20: (color online) Real part of the dimensionless Green's function of $\mathcal{O}_{S C C}$ : $\operatorname{Re} \hat{g}_{S C C}=D_{F}^{2} / T_{K} \operatorname{Re} \mathcal{G}_{S C C}$ as a function of $\omega / T_{K}$ for different values of $K_{R}$.

way this method redistributes spectral weights. This is based on the observation that the slope of the logarithm gets smaller if we increase the number of multiplets kept. These curves also collapse to a single universal curve as a function of $\omega / T_{h}$, as shown in the lower part of Fig. 22,

Finally, in Fig. 23, we show the numerically obtained spectral function and the corresponding dimensionless susceptibility of the non-composite superconductor, $\mathcal{O}_{S C}=f_{0,1, \uparrow}^{\dagger} f_{0,2, \downarrow}^{\dagger}-f_{0,1, \downarrow}^{\dagger} f_{0,2, \uparrow}^{\dagger}$. Clearly, this spectral function displays no plateau below $T_{K}$, but it exhibits a linear in $\omega$ behavior below $T_{K}$, and correspondingly, the susceptibility $\operatorname{Re} \chi_{S C}$ remains finite for $\omega \rightarrow 0$ even in the absence of anisotropy and an external magnetic field, i.e., at the $2 \mathrm{CK}$ fixed point.

This implies that, although its charge and spin quantum numbers would allow it, the expansion of this operator does not contain the scaling operator $\phi_{\Delta}^{\tau \tau^{\prime}}$. This may be due to the difference in the Ising quantum numbers, which we did not identify. Thus the dimension of the highest-weight scaling operator that appears in the

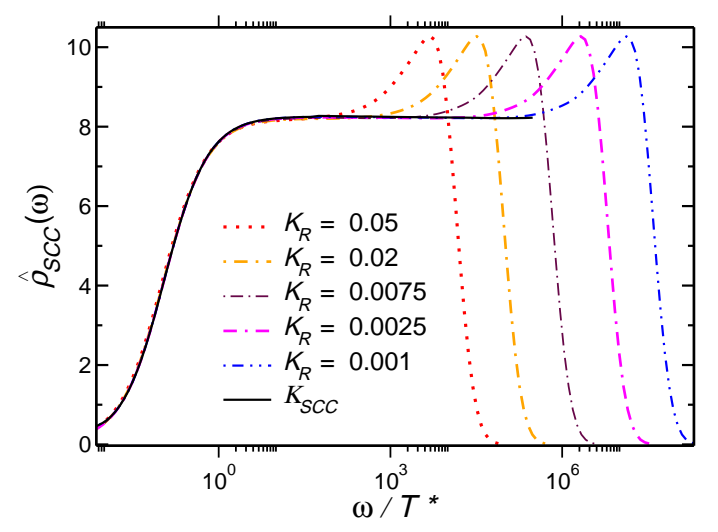

FIG. 21: (color online) Universal collapse of $\hat{\varrho}_{S C C}$ to the scaling curve $\mathcal{K}_{S C C}$ as a function of $\omega / T^{*}$ for sufficiently small, non-zero values of $K_{R}$.
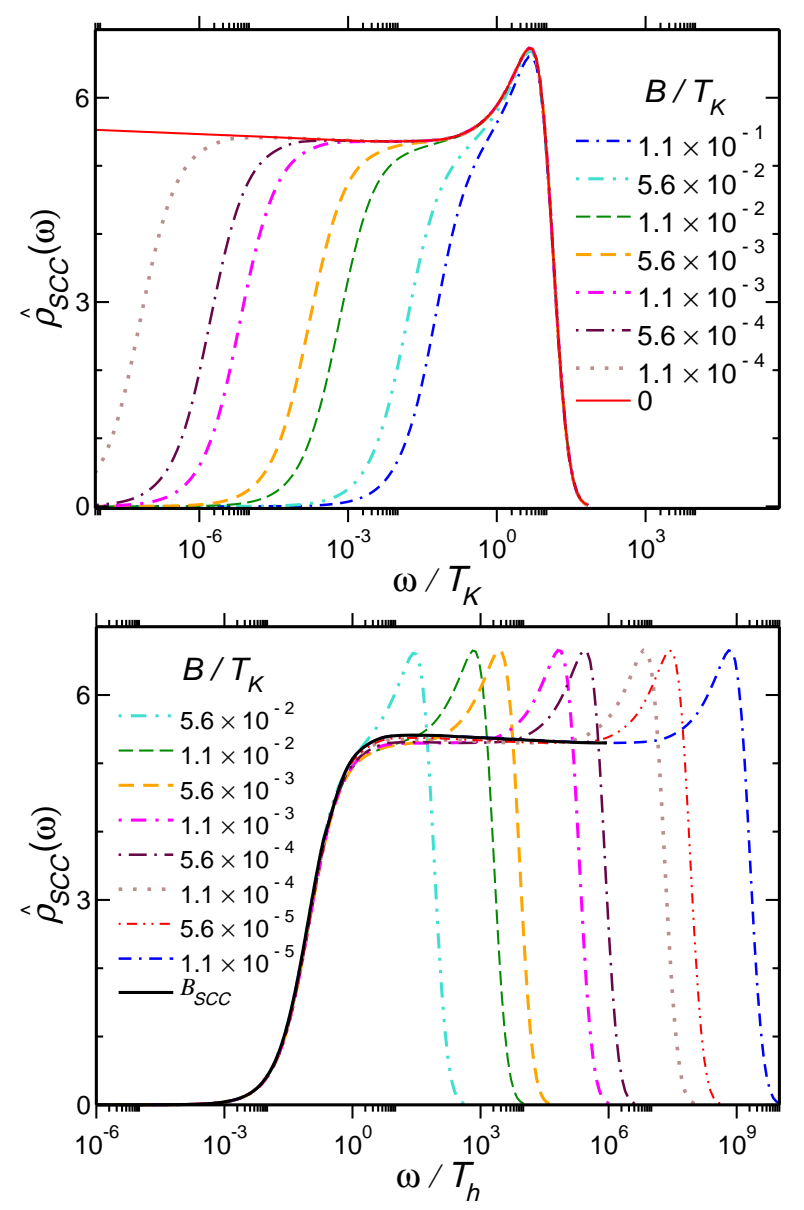

FIG. 22: (color online) Top: Dimensionless spectral function $\hat{\varrho}_{S C C}=-\left(D_{F}^{2} / \pi T_{K}\right) \operatorname{Im} \chi_{S C C}=-\left(D_{F}^{2} / \pi T_{K}\right) \operatorname{Im} \mathcal{G}_{S C C}$ of the composite superconductor operator $\mathcal{O}_{S C C}$ for different values of $B$, as a function of $\omega / T_{K}$. Bottom: Universal collapse of $\hat{\varrho}_{S C C}$ to the scaling curve $\mathcal{B}_{S C C}$ for sufficiently small, non-zero values of $B$ as a function of $\omega / T_{h}$.

expansion of $\mathcal{O}_{S C}$ is $x=1$ and not $1 / 2$, as one would naively expect based upon a simple comparison of quantum numbers. Turning on a small anisotropy or magnetic field does not influence substantially the spectral properties of the corresponding Green's function, either.

\section{CONCLUSIONS}

In the present paper we gave a detailed discussion of the spectral properties of the two-channel Kondo model. We analyzed the properties of the correlation functions of various local operators in the presence of a channel anisotropy and an external magnetic field. In particular, we studied numerically and analytically the correlation functions of the local fermions, $f_{\alpha, \sigma} \equiv f_{0, \alpha, \sigma}$, the components of the impurity spin, $\vec{S}$, the local superconductivity operator, $\mathcal{O}_{S C} \equiv f_{1}^{\dagger} i \sigma_{y} f_{2}^{\dagger}$, and the composite superconductor operator, $f_{1}^{\dagger} \vec{S} \vec{\sigma} i \sigma_{y} f_{2}^{\dagger}$. The selection of these op- 

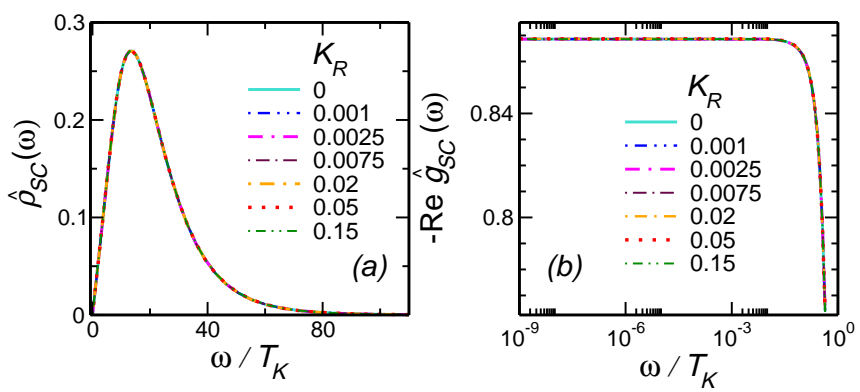

FIG. 23: (color online) (a) Dimensionless spectral function of $\mathcal{O}_{S C}: \hat{\varrho}_{S C}=D_{F} \varrho_{S C}$ as a function of $\omega / T_{K}$ for different values of $K_{R}$, and (b) the real part of its dimensionless Green's function: $\operatorname{Re} \hat{g}_{S C}=D_{F} \operatorname{Re} \mathcal{G}_{S C}$.

erators was partially motivated by conformal field theory, which tells us the quantum numbers and scaling dimensions of the various scaling operators at the two-channel Kondo fixed point ${ }^{28}$ There are, however, many operators that have quantum numbers identical with the scaling fields. Here we picked operators having the right quantum numbers, and at the same time having the largest possible scaling dimension at the free fermion fixed point, where $J_{1}, J_{2} \rightarrow 0$. These are the operators, whose spectral functions are expected to have the largest spectral weight at small temperatures (among those having the same quantum numbers), and which are therefore the primary candidates for an order parameter, when a lattice of 2CK impurities is formed, as is the case in some Uranium and Cerium-based compounds. The operators above are, of course, also of physical interest on their own: the spectral function of $f_{\alpha, \sigma}$ is related to the tunneling spectrum into the conduction electron see at the impurity site, the Green's function of $\vec{S}$ is just the dynamical spin susceptibility that can be measured under inelastic neutron scattering, and finally the local superconducting operators are candidates for superconducting ordering in heavy fermion materials. We remark that, in the electron-hole symmetrical case, the other components of the operator multiplet that contains the composite superconducting order parameter $O_{S C C}$ would correspond to a composite channel-mixing charge density ordering. Of course, the susceptibilities of this operator has the same properties as that of $\chi_{S C C}(\omega)$.

In addition to these operators, there are two more operators of possible interest: the so-called composite Fermion's Green's function is related to the $T$-matrix, $T(\omega)$ that describes the scattering properties off a twochannel impurity (or the conductance through it in case of a quantum dot), and was already studied in detail in Ref ${ }^{24}$. A further candidate is the channel anisotropy operator. This has also a logarithmically divergent susceptibility, and would also be associated with a composite orbital ordering in case of a two-channel Kondo lattice system. However, the spectral properties of this latter operator are so similar to those of the composite superconductor that we have decided no to show data about
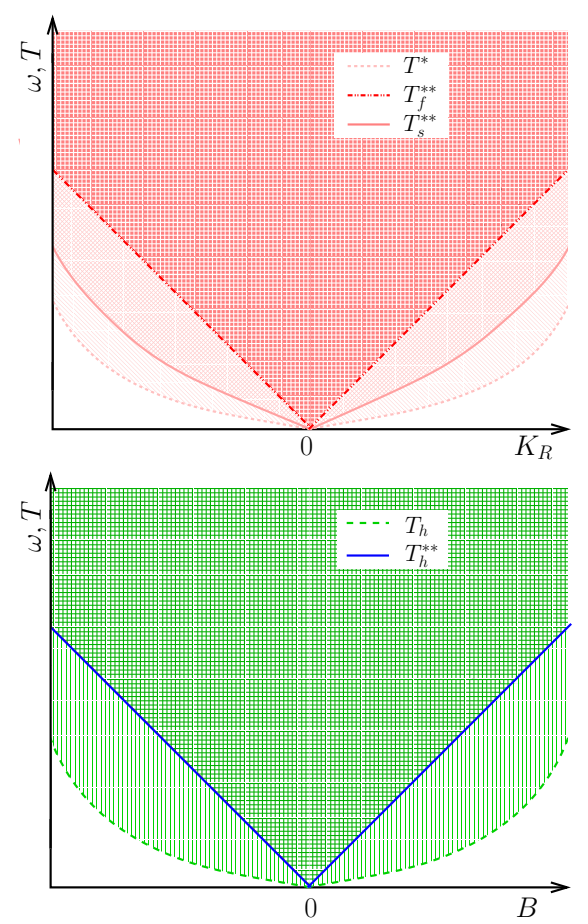

FIG. 24: (color online) Top: Sketch of the various 2CK scaling regimes in the presence of channel anisotropy for the local fermions bounded by $T_{f}^{* *}$ from below and for the spin bounded by $T_{s}^{* *}$, the crossover scale $T^{* *}$ is also indicated. Bottom: Sketch of the $2 \mathrm{CK}$ scaling regime for the susceptibilities of the highest-weight fields bounded by $T_{h}^{* *}$, and the crossover scale $T_{h}$ besides.

them.

For the numerical calculations we used a flexible DMNRG method, where we exploited the hidden charge $\mathrm{SU}(2)$ symmetries ${ }^{45}$ as well as the invariance under spin rotations to obtain high precision data. To identify the scaling operators in this case, we reconstructed the boundary conformal field theory of Affleck and Ludwig for this symmetry classification. We then established the scaling properties of the various dynamical correlation functions and identified the corresponding universal cross-over functions and their asymptotic properties, based upon simple but robust scaling arguments. In this way, universal scaling functions describing the cross-over from the two-channel Kondo fixed point to the single channel Kondo fixed point (for $J_{1} \neq J_{2}$ ) and to the magnetically polarized fixed point (for $B \neq 0$ ) have been introduced, which we then determined numerically. We emphasize again that presently these universal cross-over functions can only be determined through the application of DM-NRG, and in fact, for the scaling curves in the presence of a magnetic field the application of the DM-NRG method was absolutely necessary.

Our numerical calculations confirmed all our analytical expectations, and they confirmed that actually, in the presence of an applied magnetic field, or channel anisotropy, the two-channel Kondo scaling regime is 
rather restricted, and it may also depend on the physical quantity considered. In Fig 24 we sketched the regimes where the pure two-channel Kondo behavior can be observed. Notice that in the presence of anisotropy the two-channel Kondo scaling regime of the spin susceptibility has a boundary that differs from the boundary of the two-channel Kondo scaling regime of the $T$-matrix.

Some of the spectral functions show rather remarkable features: In a magnetic field, e.g., the spectral function of the composite fermion, $F_{\alpha, \downarrow}^{\dagger}$ shows a universal peak at a frequency $\omega=T_{h}$. This peak corresponds to spinflip excitations of the impurity spin at the renormalized magnetic field. Remarkably, this peak is accompanied by a dip of the same size at the same frequency for spin down electrons. This dip is actually very surprising and is much harder to explain. Similar features appear but with opposite sign in the local fermions' spectral functions. Even more surprisingly, this resonant feature is completely absent in the spectral function of the spin operators, $S^{ \pm}$.

One of the interesting results of our numerical analysis was that only the composite superconductor $\mathcal{O}_{S C}$ has a logarithmically divergent susceptibility. This is thus the primary candidate for superconducting ordering for a $2 \mathrm{CK}$ lattice system. We remark here that while for a single impurity the superconducting susceptibility seems to have a rather small amplitude, $\operatorname{Re} \chi_{S C} \sim$ $T_{K} / D_{F}^{2} \ln \left(T_{K} / T\right)$, in a lattice model the mass of the carriers is also renormalized, and therefore the bandwidth is expected to get renormalized as $D_{F} \rightarrow T_{K} \stackrel{5}{\underline{5}} \mathrm{As}$ a result, the corresponding susceptibility can be rather large, and drive, in principle, a superconducting instability. Interestingly, although the results are still somewhat controversial, $\stackrel{43}{ }$ in the two-channel Kondo lattice these local superconducting correlations do not seem to induce a superconducting transition $\stackrel{47}{ }$ This may be, however, an artifact of the standard two-channel Kondo lattice model, which does not account properly for the orbital and band structure of an $f$-electron material. 44 We believe, that in a more realistic lattice of two-channel Kondo impurities a composite superconducting order develops, similar to the one suggested in Ref. 44. However, DMFT + DM-NRG calculations would be needed to confirm this belief.

Acknowledgement: We are especially grateful to L. Borda for making his code available for the Hilbert transformations and for the lot of valuable discussions. Useful comments on the manuscript from Z. Bajnok and I. Cseppkövi are highly appreciated. This research has been supported by Hungarian grants OTKA Nos. NF061726, T046267, T046303, D048665, NK63066.

\section{APPENDIX A: SCALING PROPERTIES OF TWO-POINT FUNCTIONS}

In this appendix, we discuss the scaling properties of various scaling functions. Essentially, we use the generalized Callan-Symanzik equations. For the sake of simplicity, let us first focus on the retarded Green's function of the $z$-component of the operator $\phi_{s}$,

$$
\mathcal{G}(t, \mathcal{H}) \equiv-i\left\langle\left[\phi_{s}^{z}(t), \phi_{s}^{z}(0)\right]\right\rangle_{\mathcal{H}} \theta(t)
$$

and its Fourier transform, $\mathcal{G}(\omega, T)$. Let us investigate the scaling properties of this function in the absence of magnetic field. From the fact that $\phi_{s}$ is the field conjugate to the external "magnetic field", $h$, and that the partition function (generating function) must be scale invariant under the renormalization group, we easily get the following differential equation

$$
D \frac{\partial \mathcal{G}}{\partial D}+\sum_{\mu} \beta_{\mu} \frac{\partial \mathcal{G}}{\partial u_{\mu}} u_{\mu}+\left(2 \beta_{h}-1\right) \mathcal{G} \approx 0
$$

with $u_{\mu}$ a shorthand notation for the dimensionless couplings, $\left\{u_{\mu}\right\}=\{\kappa, \lambda, \ldots\}$ that occur in $\mathcal{H}$, and $\beta_{\mu}$ the corresponding $\beta$-functions,

$$
\frac{\mathrm{d} \ln u_{\mu}}{\mathrm{d} x}=\beta_{\mu}\left(\left\{u_{\nu}\right\}\right),
$$

with $x=-\ln (D)$ the scaling variable. In the vicinity of the two-channel Kondo fixed point the $\beta$-functions just assume their fixed point value, which are just the renormalization group eigenvalues, $y_{\mu}=d-x_{\mu}$, with the dimension $d=1$, since all operators are local and live in time only. Since, for $\phi_{s}$ we have $y_{h}=1 / 2$, in the close vicinity of the two-channel Kondo fixed point we obtain

$$
\frac{\mathrm{d} \mathcal{G}}{\mathrm{d} D} \approx 0
$$

One can also easily show that

$$
D \frac{\mathrm{d} \mathcal{G}}{\mathrm{d} D}=-\omega \frac{\mathrm{d} \mathcal{G}}{\mathrm{d} \omega}
$$

These relations imply that, $\mathcal{G}(\omega, T, D)$ is scale invariant, and is only a function of $\omega / D$ and $T / D$. Clearly, similar equations hold for the correlation functions of all operators with dimension $1 / 2$. Furthermore, the above scaling property can easily be modified for operators having dimensions $y_{\mu} \neq 1 / 2$.
${ }^{1}$ For a recent review see P. A. Lee, From high temperature superconductivity to quantum spin liquid: progress in strong correlation physics, submitted for Report of Progress in Physics (2007).

2 E. W. Carlson et al., Concepts in High Temperature Super- conductivity in The Physics of Conventional and Unconventional Superconductors, Vol. II. ed. K. H. Bennemann and J. B. Ketterson, Springer-Verlag (2004).

3 H. von Löhneysen, A. Rosch, M. Vojta, P. Wölfle, Rev. Mod. Phys. 79, 1015 (2007). 
${ }^{4}$ P. Coleman, Heavy Fermions: electrons at the edge of magnetism, in Handbook of Magnetism and Advanced Magnetic Materials, J. Wiley and Sons (2007).

5 For a review see D. L. Cox, A. Zawadowski, Adv. in Phys., 47, 599 (1998).

${ }^{6}$ Q. Si et al., Nature 413, 804-808 (2001).

7 M. Vojta, Phil. Mag. 86, 1807 (2006).

8 M. Vojta, Rep. Prog. Phys. 66, 2069 (2003).

9 J. M. Luttinger, J. Math. Phys. 4, 1154 (1963).

10 M. Bockrath et al., Nature 397, 598 (1999).

11 H. Ishii et al., Nature 426, 540 (2003).

12 P. M. Singer et al., Phys. Rev. Lett. 95, 236403 (2005).

13 B. Dóra, M. Gulácsi, F. Simon and H. Kuzmany, Phys. Rev. Lett. 99, 166402 (2007).

14 M. Milovanović et al., Phys. Rev. Lett. 63, 82 (1989).

15 V. Dobrosavljević et al., Phys. Rev. Lett. 69, 1113 (1992).

$16 \mathrm{Ph}$. Nozières and A. Blandin, J. Phys. Paris, 41, 193 (1980).

17 S. Katayama, S. Maekawa and H. Fukuyama, J. Phys. Soc. Jpn. 50, 694 (1987).

18 J. von Delft et al., Ann. Phys. 263, 1 (1998).

19 T. Cichorek et al., Phys. Rev. Lett. 94, 236603 (2005).

20 Y. Oreg and D. Goldhaber-Gordon, Phys. Rev. Lett. 90, 136602 (2003).

21 R. M. Potok et al., Nature 446, 167 (2007).

22 M. Pustilnik, Phys. Rev. B, 69, 115316 (2004).

${ }^{23}$ F. B. Anders, E. Lebanon and A. Schiller, Phys. Rev. B 70, 201306 (2004).

24 A. I. Tóth, L. Borda, J. von Delft and G. Zaránd, Phys. Rev. B 76, 155318 (2007).

25 Ph. Nozières, J. Low Temp. Phys. 17, 31 (1974).

${ }^{26}$ N. Andrei and C. Destri, Phys. Rev. Lett. 52, 364 (1984).

27 A. M. Tsvelick, P. B. Wiegmann, J. Stat. Phys. 38, 125 (1985).

28 I. Affleck, A. W. W. Ludwig, Nucl. Phys. B 352, 849 (1991); ibid 360, 641 (1991), I. Affleck et al., Phys. Rev. B 45, 7918 (1991).

${ }^{29}$ K. G. Wilson, Rev. Mod. Phys. 47, 773 (1975).

30 H. B. Pang and D. L. Cox, Phys. Rev. B 44, 9454 (1991).
31 K. Vladár, A. Zawadowski, G. T. Zimányi, Phys. Rev. B, 37, 2001 (1988); ibid 37, 2015 (1988)

32 V. J. Emery and S. Kivelson, Phys. Rev. B 47, 10812 (1992).

33 J. Gan, N. Andrei and P. Coleman, Phys. Rev. Lett. 70, 686 (1993).

34 G. Zaránd and K. Vladár, Phys. Rev. Lett. 76, 2133 (1996).

35 D. L. Cox and A. E. Ruckenstein, Phys. Rev. Lett. 71, 1613 (1993).

36 L. Borda et al., Phys. Rev. B 75, 205125 (2005).

37 I. Affleck and A. W. W. Ludwig, Phys. Rev. B 48, 7297 (1993).

38 J. Kroha, P. Wölfle and T. A. Costi, Phys. Rev. Lett. 79, 261 (1997).

39 A. M. Sengupta, A. Georges, Phys. Rev. B 49, 10020 (1994).

40 S. Suzuki, O. Sakai and Y. Shimizu, Solid State Comm. 104429 (1997).

41 W. Hofstetter, Phys. Rev. Lett. 85, 1508 (2000); R. Peters, T. Pruschke, F. B. Anders, Phys. Rev. B 74, 245114 (2006); A. Weichselbaum, J. von Delft, Phys. Rev. Lett. 99, 076402 (2007).

42 A. I. Tóth et al., unpublished.

43 M. Jarrell et al., Phys. Rev. Lett. 77, 1612 (1996).

44 N. Andrei et al., J. Phys. Cond. Mat., 10, L239 (1998); P. Coleman et al., Phys. Rev. B 60, 3608 (1999).

45 B. A. Jones, C. M. Varma and J. W. Wilkins, Phys. Rev. Lett. 61, 125 (1987).

46 T. Costi, Phys. Rev. Lett. 85, 1504 (2000).

47 F. B. Anders, M. Jarrell and D. L. Cox, Phys. Rev. Lett. 78, 2000 (1997).

48 M. Garst et al., Phys. Rev. B 72, 205125 (2005).

${ }^{49}$ For further theoretical studies see Ref.-s $22,23.24$.

50 Throughout the paper we use units of $\hbar=k_{B}=v_{F}=1$.

51 Throughout this paper we discuss only retarded Green's functions. The other Green's functions are related to them by simple analytic relations in equilibrium. 